\title{
Patterns of somatic structural variation in human cancer genomes
}

https://doi.org/10.1038/s41586-019-1913-9

Received: 22 September 2017

Accepted: 18 November 2019

Published online: 5 February 2020

Open access

Mutations that arise in somatic cells are the driving force of cancer development. Structural variation-in which genomic rearrangement acts to amplify, delete or reorder chromosomal material at scales that range from single genes to entire chromosomes-is an especially important class of somatic mutation. Previous analyses of both cancer and germline genomes have enabled the description of several distinctive patterns of structural variants ${ }^{1-7}$, and hypotheses about the underlying basis of several of these patterns have been proposed on the basis of their clustering, orientation and associated copy-number changes. Hypothesis-driven in vitro studies are now beginning to reveal some of the mechanistic processes that generate these structures ${ }^{9-13}$, and generate further predictions that can be assessed in the genomic data. However, the landscape of structural variation in human cancer remains incompletely mapped and there are many complex structures that elude formal description.

The PCAWG Consortium aggregated whole-genome sequencing data from 2,658 cancers across 38 tumour types, generated by the ICGC and TCGA projects. These sequencing data were aligned to the human genome (reference build hs37d5) and analysed with standardized, highaccuracy pipelines to call somatic and germline variants of all classes ${ }^{8}$. Here, we analyse the patterns and signatures of structural variants across the PCAWG data. We propose a working classification scheme that encompasses known and newly identified classes of structural variants. We develop methods for annotating the observed structural variants in a given cancer genome, identifying a class of replication-based rearrangement processes that generate clusters of several structural variants. We explore the size, activity and genome-wide distribution of classifiable structural variant types across the cohort, using signature analysis to define how they correlate within patients. Other papers produced by PCAWG address complementary aspects of structural variants, including inference of positive selection acting on recurrently rearranged regions of the genome ${ }^{14}$, how structural variants affect the transcriptome $^{15}$ and chromosome topology ${ }^{16}$, patterns of somatic retrotransposition $^{17}$ and distribution of chromothripsis across cancer types ${ }^{18}$.

${ }^{1}$ Cancer Genome Project, Wellcome Trust Sanger Institute, Hinxton, UK. ${ }^{2}$ Totient Inc, Cambridge, MA, USA. ${ }^{3}$ The Broad Institute of Harvard and MIT, Cambridge, MA, USA. ${ }^{4}$ Bioinformatics and

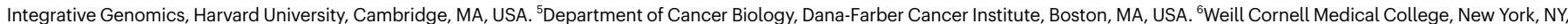
USA. ${ }^{7}$ European Molecular Biology Laboratory, Genome Biology Unit, Heidelberg, Germany. ${ }^{8}$ Department of Molecular Biology, Rosenstiel Basic Medical Sciences Research Center, Brandeis University, Waltham, MA, USA. ${ }^{9}$ New York Genome Center, New York, NY, USA. ${ }^{10} \mathrm{~A}$ list of members and their affiliations appears at the end of the paper. ${ }^{11}$ Biotech Research \& Innovation Centre

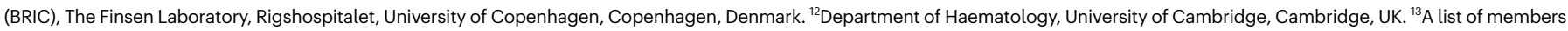
and their affiliations appears online. ${ }^{920}$ These authors contributed equally: Yilong Li, Nicola D. Roberts, Jeremiah A. Wala, Ofer Shapira. *e-mail: joachim.weischenfeldt@bric.ku.dk:

rameen_beroukhim@dfci.harvard.edu; pc8@sanger.ac.uk 


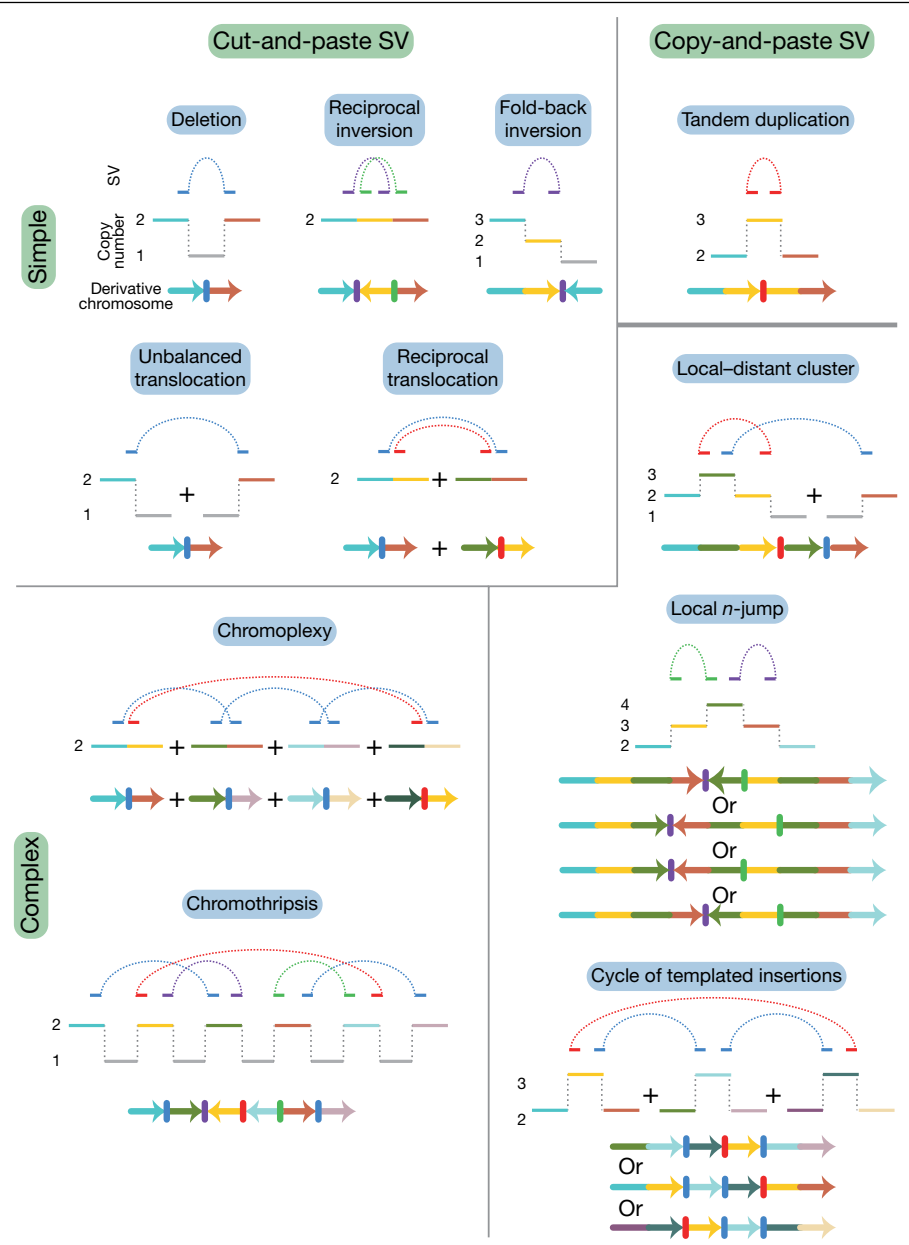

Fig. 1 | Classification of structural variants in cancer genomes. Schematics of major structural-variant (SV) classes, grouped according to whether they are simple or complex and arise through cut-and-paste or copy-and-paste processes. Each schematic comprises three parts. The top segment shows dotted arcs for each rearrangement junction that joins two chromosomal segments together. The middle segment shows the copy number of genomic segments that are involved. The bottom segment shows the configuration of the final derivative chromosome that results from the structural variant; the colour of the segments corresponds to the colour of that segment in the copynumber schematic. + indicates the different derivative chromosomes created for some of the classes: that is, the structural variants are not phased to a single derivative.

\section{Classification of structural variants}

A 'structural variant' manifests as a 'junction' between two 'breakpoints' in the genome (terms in inverted commas here and below refer to those defined in the glossary in Extended Data Table 1). Generally, there will be a change in copy number across a given breakpoint if only one side of the break is rescued by a structural variant; if both sides of a doublestranded DNA break are rescued, a 'reciprocal' or 'balanced' structural variant will result, without substantial copy-number change. We sometimes observe 'clusters of structural variants' in which several breakpoints occur close together, in time or in genomic space-usually both. Such spatial and/or temporal proximity generally, but not always, implies that the structural variants within a cluster are mechanistically linked. Clusters can be 'phased' (in which case all structural variants in the cluster resolve to a single derivative chromosome) or 'unphased', in which case the structural variants are carried on different derivative chromosomes. An example of the latter is a reciprocal translocation that results in two derivative chromosomes, each with a single interchromosomal breakpoint junction (Fig. 1).
We recognize distinct 'classes of structural variant' from the orientation of the two segments at the junction and associated copy-number changes (Fig. 1, Supplementary Fig. 1). Some classes of structural variant (such as isochromosomes and rearrangements between extended, highly homologous sequences) are difficult to detect with short-read sequencing data; these classes are not considered further here. We propose categorizing classes of structural variant across two facets: the number of breakpoints involved (simple or complex) and by whether the patterns are likely to arise from 'cut-and-paste' or 'copy-and-paste' rearrangement processes. A cut-and-paste process generates a cluster of structural variants consistent with reshuffling or loss of extant genomic segments, and a copy-and-paste process is one in which copies of genomic 'templates' are newly replicated or synthesized and inserted during the rearrangement process. Deletions, reciprocal inversions, unbalanced translocations and reciprocal translocations are examples of simple cut-and-paste structural variants, as they can be reconstructed from the incorrect religation of chromosomal breaks. Tandem duplications are simple copy-and-paste structural variants, as they arise through the local insertion of a newly generated extra copy of a genomic template.

More-complex cut-and-paste processes that produce structural variants also occur in cancer. 'Breakage-fusion-bridge' events result from cycles of DNA breakage, end-to-end sister chromatid fusions, mitotic bridges and further DNA breakage. These events manifest as one or a few proximate, inverted breakpoint junctions with associated copy-number change, which we call ' fold-back inversions ${ }^{1,2,19}$ (Fig. 1). 'Chromoplexy ${ }^{5,20}$ - which is particularly frequent in prostate cancersresults from several simultaneous double-stranded DNA breaks in several chromosomes that are rejoined incorrectly, leading to balanced chains of rearrangements. 'Chromothripsis's, in which chromosome shattering and rearrangement occur in a single catastrophic event ${ }^{9,21}$, leads to a pattern of oscillating copy-number changes and localized clustering of tens to hundreds of breakpoints ${ }^{22}$.

In the germline, more-complex copy-and-paste classes of structural variant have previously been described, which involve small duplications and triplications and are thought to arise from the stalling of the replication fork leading to template switching ${ }^{4,23,24}$. Here we describe a wide range of complex copy-and-paste types of somatic structural variant that occur in human cancers, and that are typically characterized by copy-number gains and frequent inverted rearrangements.

\section{Annotation of structural-variant classes}

We analysed 2,559 whole cancer genomes across 38 tumour types (alongside matched germline DNA) that passed the most stringent PCAWG quality-control criteria: 1 or more somatic structural variants were detected in 2,429 tumours $^{8}$. As described in an accompanying Article $^{8}$, structural variants were identified using aberrantly mapping and/or split reads in paired-end sequencing data ${ }^{25}$. We used four somatic structural-variant callers ${ }^{20,25-27}$, and the final structural-variant dataset comprised events that were returned by $\geq 2$ callers, merged by a graph-based consensus method $^{8}$. We consider only somatically acquired structural variants in this analysis, and exclude somatic retrotransposition events. Validation of structural-variant calls was undertaken using both manual inspection and pull-down with resequencing of breakpoints. With these approaches, we estimate the sensitivity of the consensus structural-variant call set to be $90 \%$ for true calls generated by any 1 of the 4 callers; specificity was estimated as $97.5 \%^{8}$. A mean of 3.22 algorithms of the 4 that we used called each structural variant in the consensus set genome-wide, and this differed little across repetitive elements: the mean for short interspersed nuclear elements was 3.22, and the mean for long interspersed nuclear elements was 3.21.

Because the structural variants from a given cancer are often highly clustered, we grouped rearrangements into clusters on the basis of the 


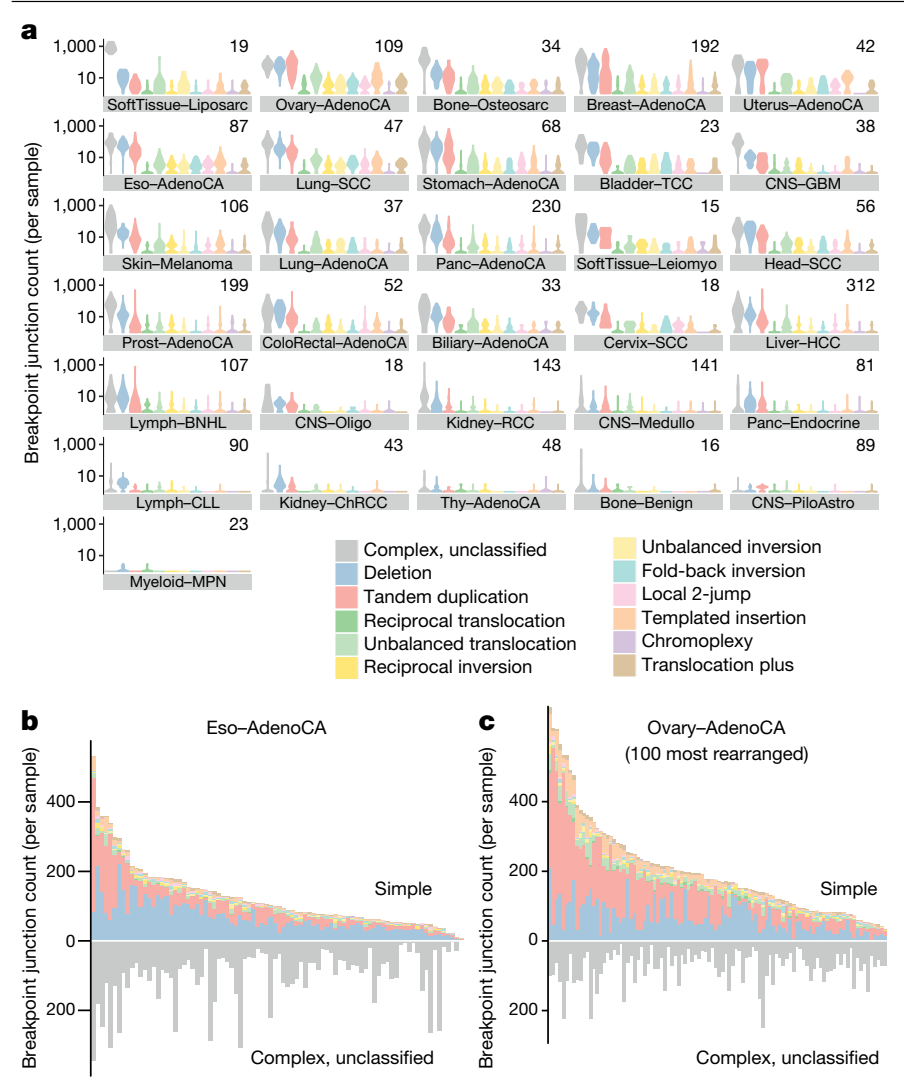

Fig. 2 | Frequency of structural-variant classes across tumour types. a, Violin plots of density of classified structural-variant categories across patients within each histology group. Tumour type panels are sorted in descending order of the average number of structural-variant breakpoints per sample. Within each tumour type, the frequency distribution ( $y$ axis) of different structural-variant categories ( $x$ axis) across patients is shown as a density: regions of highest density have the greatest width of shaded area. In each panel, the number of patients is indicated at the top right. AdenoCA, adenocarcinoma; BNHL, B-cell non-Hodgkin lymphoma; ChRCC, chromophobe renal cell carcinoma; CLL, chronic lymphocytic leukaemia; CNS, central nervous system; GBM, glioblastoma; HCC, hepatocellular carcinoma; leiomyo, leiomyosarcoma; medullo, medulloblastoma; MPN,

myeloproliferative neoplasm; eso, oesophageal; oligo, oligodendrocytic; panc, pancreatic; piloastro, pilocytic astrocytoma; prost, prostate; RCC, renal cell carcinoma; sarc, sarcoma; SCC, squamous cell carcinoma; TCC, transitional cell carcinoma; thy, thyroid. b, Per-sample counts of complex (bottom) and classified (top) structural-variant breakpoint junctions for oesophageal adenocarcinoma. c, Per-sample counts of complex (bottom) and classified (top) structural-variant breakpoint junctions for ovarian adenocarcinoma.

proximity of breakpoints, the overall number of events in that genome and the size distribution of these events (Supplementary Methods). Essentially, a particular cluster contains structural variants that are significantly closer together than expected by chance, given the overall number and orientation of structural variants in that patient. Alongside the clustering, we computed an in silico library of all possible genomic configurations that result from sequential simple structural variants (deletions, tandem duplications, inversions, translocations, and chromosome duplications or losses), to a depth of five rearrangements. We could then compare the genomic configuration of each observed cluster of structural variants against the library to determine how it might have arisen.

This methodology has the advantage that breakpoint junctions are classified according to the wider genomic context in which they occur. This means that, for example, true deletions will be identifiably different from breakpoint junctions that happen to have a deletion-type orientation but arise within (for instance) a chromothripsis event of markedly different mechanism and properties. Over half the breakpoint junctions that we observed arise within clusters of several or many structural variants (Fig. 2a): removing these junctions from the catalogues of true deletions, tandem duplications and inversions enables a more-precise description of the properties of simple structural variants.

Among the classes of simple structural variants, deletion was the most common, followed by tandem duplication and then unbalanced translocation. Reciprocal translocations and reciprocal inversions were uncommon events (Fig. 2a). There was considerable variability in the overall numbers and distribution of classes of structural variant across tumour types and across patients within a given tumour type (Extended Data Fig. 1). For example, oesophageal adenocarcinomas were characterized by many deletions and a large number of complex clustered rearrangements (Fig. 2b), and ovarian cancers often carried high numbers of tandem duplications and/or deletions with moderate numbers of unbalanced translocations (Fig. 2c).

\section{Cycles of templated insertions}

We next examined clusters that contain 2-10 structural variants. One newly identified configuration consisted of several segments of copynumber gains, typically on different reference chromosomes, linked together through structural variants (Fig. 3, Extended Data Fig. 2). A sequential path through consecutive segments can be formed by following the breakpoint junctions, which suggests that each cluster represents a string of duplicated templates inserted into a single derivative chromosome, probably acquired concurrently. Although it is theoretically possible that the structural variants in such clusters are not phased on the same derivative chromosome or do not occur concurrently, we think this is unlikely for several reasons. First, we found examples of RNA transcripts that spliced together exons separated by two junctions in the structural-variant cluster (Supplementary Fig. 2), which suggests that they are phased on the same derivative chromosome. Second, long-read sequencing data (reported in an accompanying $\mathrm{Article}^{8}$ ) supported the phasing of structural variants that link templated insertions. Third, we found that the clonal fraction of tumour cells tended to be more similar for structural variants within these clusters than for randomly chosen structural variants in each patient (Supplementary Fig. 3), which suggests that they cooccur in evolutionary time. Fourth, the level of copy-number gain for individual segments in the cluster tended to be identical (Fig. 3, Extended Data Fig. 2).

We define three basic categories on the basis of whether or not the string of inserted segments returns to the original chromosome: we term strings of inserted segments that do not return 'chains' of templated insertions and those strings that do return 'bridges' (which leave a gap on the host chromosome) or 'cycles' (which rereplicate a segment on the host chromosome). In the PCAWG dataset overall, we observed 1,467 cycles and 1,275 bridges of templated insertions (Fig. 3a, b, Extended Data Fig. 2). In chains of templated insertions, the string of genomic segments does not return to the chromosome of departure (Fig. 3c, Extended Data Fig. 2) but it is similarly associated with copy-number gains at each templated segment. There were 285 instances of such chains in the dataset, commonly manifesting as unbalanced translocations joined through one or more intermediary templated insertions.

Most templated insertion events involve only two breakpoint junctions, but this can extend to three, four or more linked rearrangements (Extended Data Fig. 3a). The longest such event-from a cervical squamous cell cancer-had seven templated insertions strung together on an eighth host chromosome (Fig. 3c; other examples of long templated insertion events are shown in Extended Data Fig. 3). 


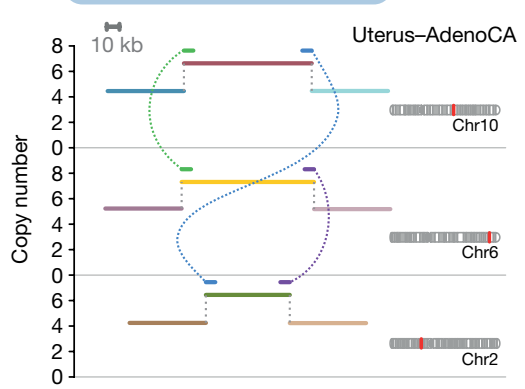

Possible derivative chromosomes:

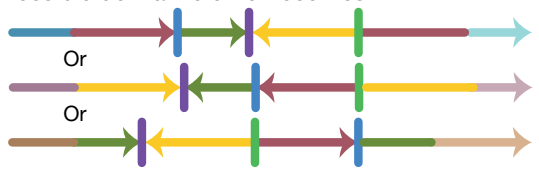

b Chain of templated insertions

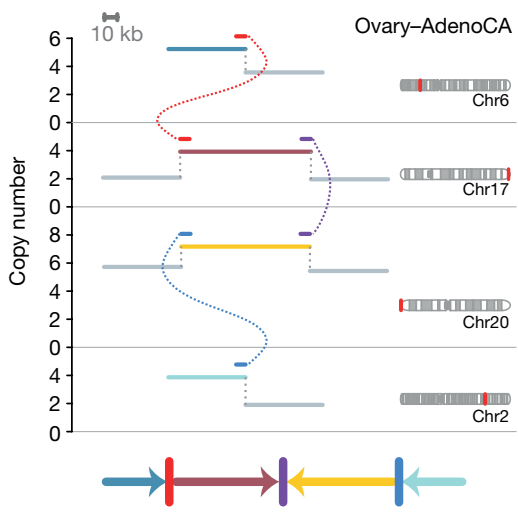

Cervix-SCC
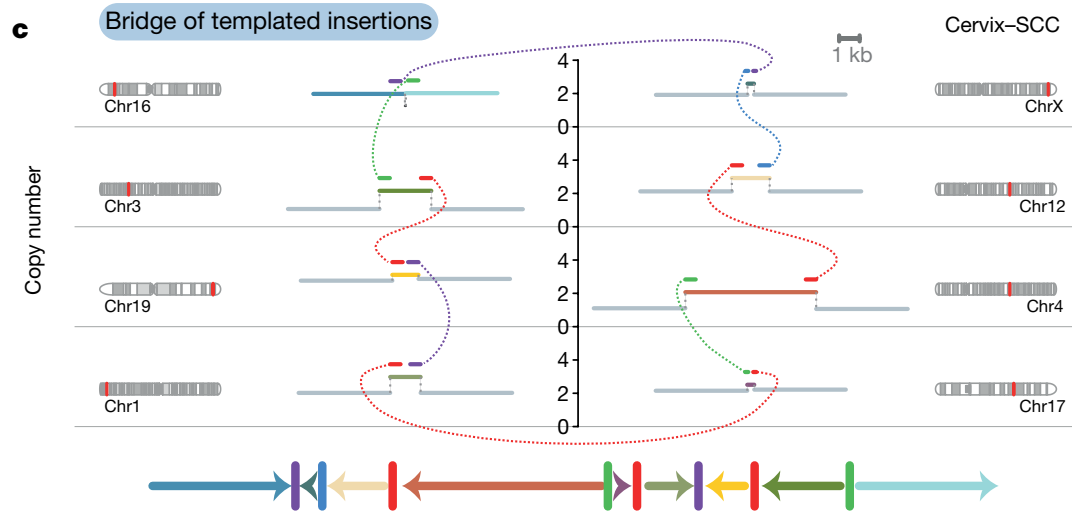

d

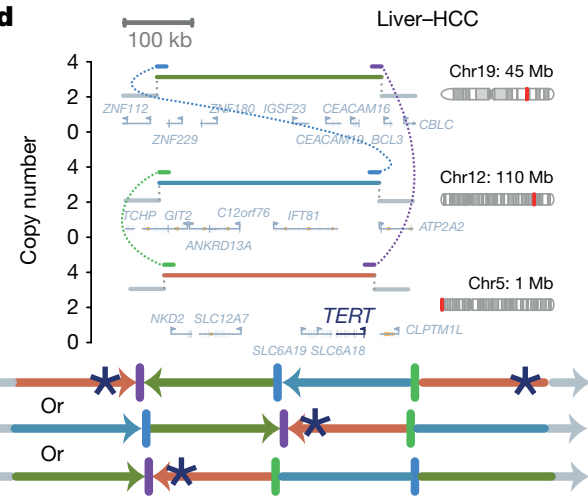

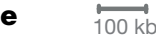
Liver-HCC

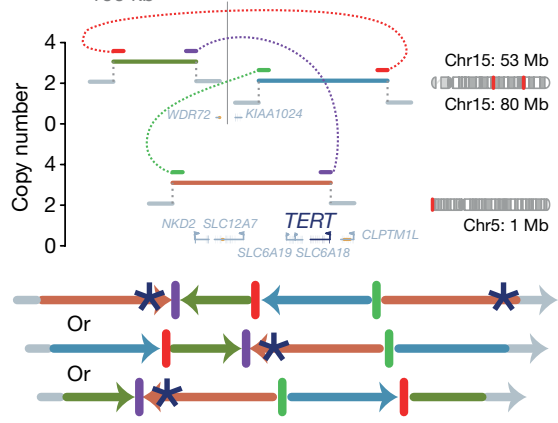

chromosome(s) that could explain the copy-number and structural-variant profile is shown below. d, e, Cycles of templated insertions that affect the TERT gene, in two hepatocellular carcinomas. KIAA1O24 is also known as MINAR1.

TERT gene and linked it to duplications of whole genes, fragments of genes or regulatory elements from elsewhere in the genome, and led to increased expression of TERT (Extended Data Fig. 4e). Thus, this particular rearrangement process is distinctive for the precision with which cancer copy-and-pastes normally disparate functional elements of its genome together without wholesale instability.

Tumour-suppressor genes were also inactivated by templated insertions (Extended Data Fig. 5). For example, among many straightforward deletions, $R B 1$ was hit by cycles of templated insertions, a templated insertion with deletion and one instance of the linked, inverted duplications detailed in 'Local $n$-jumps and local-distant clusters'. These events typically generated duplications of internal exons in $R B 1$ and/or affect $T E R T$, we find that 10 of these variants were templated insertion events (mostly cycles). All of these events duplicated the entire 
a

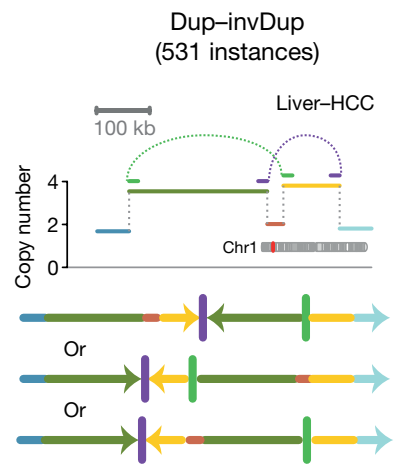

b

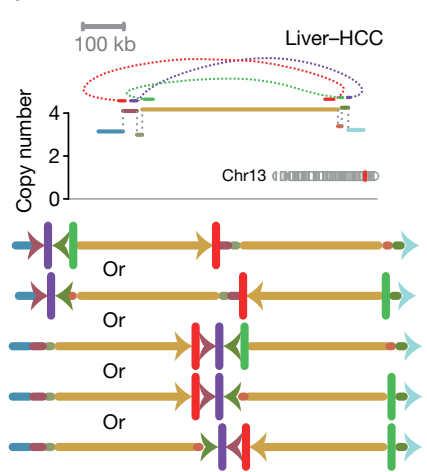

c

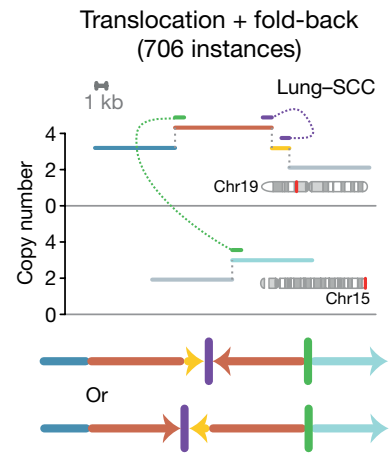

Fig. 4 | Examples of clusters of 2-5 rearrangements seen in human cancers. a, Structures created by two local rearrangements that cannot easily be explained by simple structural-variant classes (which we call local 2-jumps). The estimated copy-number profile is shown as in Fig. 1, with structural variants shown as dotted arcs linking two copy-number segments. Possible configurations of the derivative chromosome are shown below; multiple

insertions of exons from other genes, all of which presumably rendered a non-functional transcript.

\section{Local $n$-jumps and local-distant clusters}

Many clusters of $2-10$ structural variants in the dataset were confined to a single genomic region. Of those clusters that comprised two local rearrangements, some had straightforward explanations, such as nested or adjacent tandem duplications. However, many did not have a trivial explanation (Fig. 4a). These included a duplication-invertedtriplication-duplication structure that has previously been observed in germline structural variants ${ }^{24}$ (349 instances); a structure of two duplications linked by inverted rearrangements (531 instances); and structures of copy-number loss plus nearby duplication linked by inverted
Local 2-jumps
Dup-Trp-Dup

(349 instances)

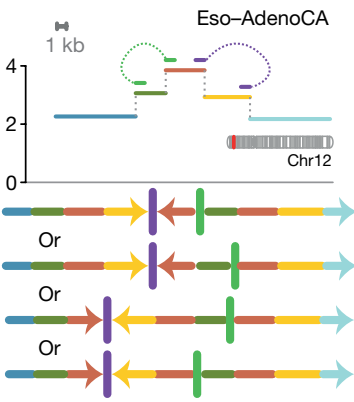

Local 3- and 4-jumps

29 instances

Breast-AdenoCA

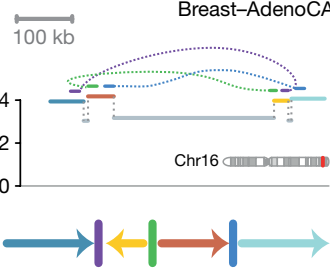

ocal-distant clusters
oss-invDup

(472 instances)

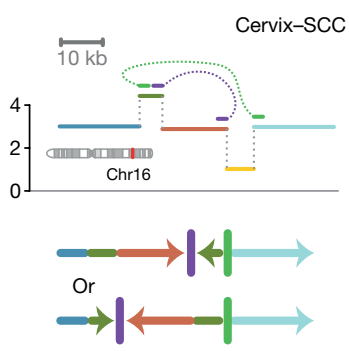

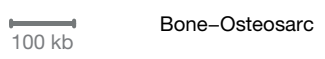

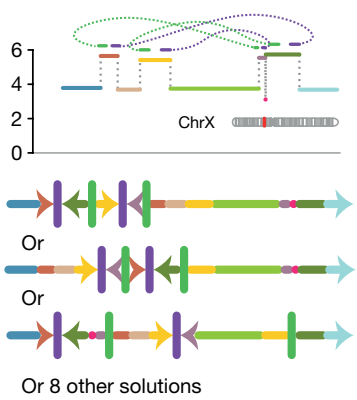
561 instances)

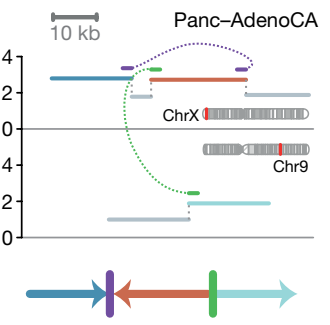

Translocation + tandem duplication (204 instances)

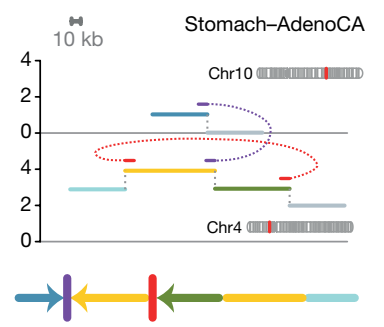

solutions are possible for each example. Dup, duplication; invDup, duplication linked by inverted rearrangement; trp, triplication. b. Structures created by 3-4 local rearrangements that cannot easily be explained by simple structuralvariant categories. $\mathbf{c}$, Structures created by one local rearrangement and one rearrangement that reaches elsewhere in the genome (local-distant clusters). rearrangements (472 instances). All of these patterns had solutions in which breakpoints were phased to a single derivative chromosome (Fig. 4a), although non-phased solutions are theoretically possible (if unlikely). Beyond clusters of two rearrangements (two-jumps), we also found examples involving three, four or more rearrangements confined to one genomic locale (Fig. 4b). All of these configurations of clusters of structural variants can be phased to a single derivative chromosome, with tightly grouped breakpoints.

Beyond clusters confined to a single genomic region, we found clusters of 2-10 structural variants that combined local jumps with rearrangements that reach into one or more distant regions of the genome (Fig. 4c). Simple examples of these events include unbalanced translocations or large deletions with a locally derived fragment inserted at the breakpoint, but there was also an extensive 

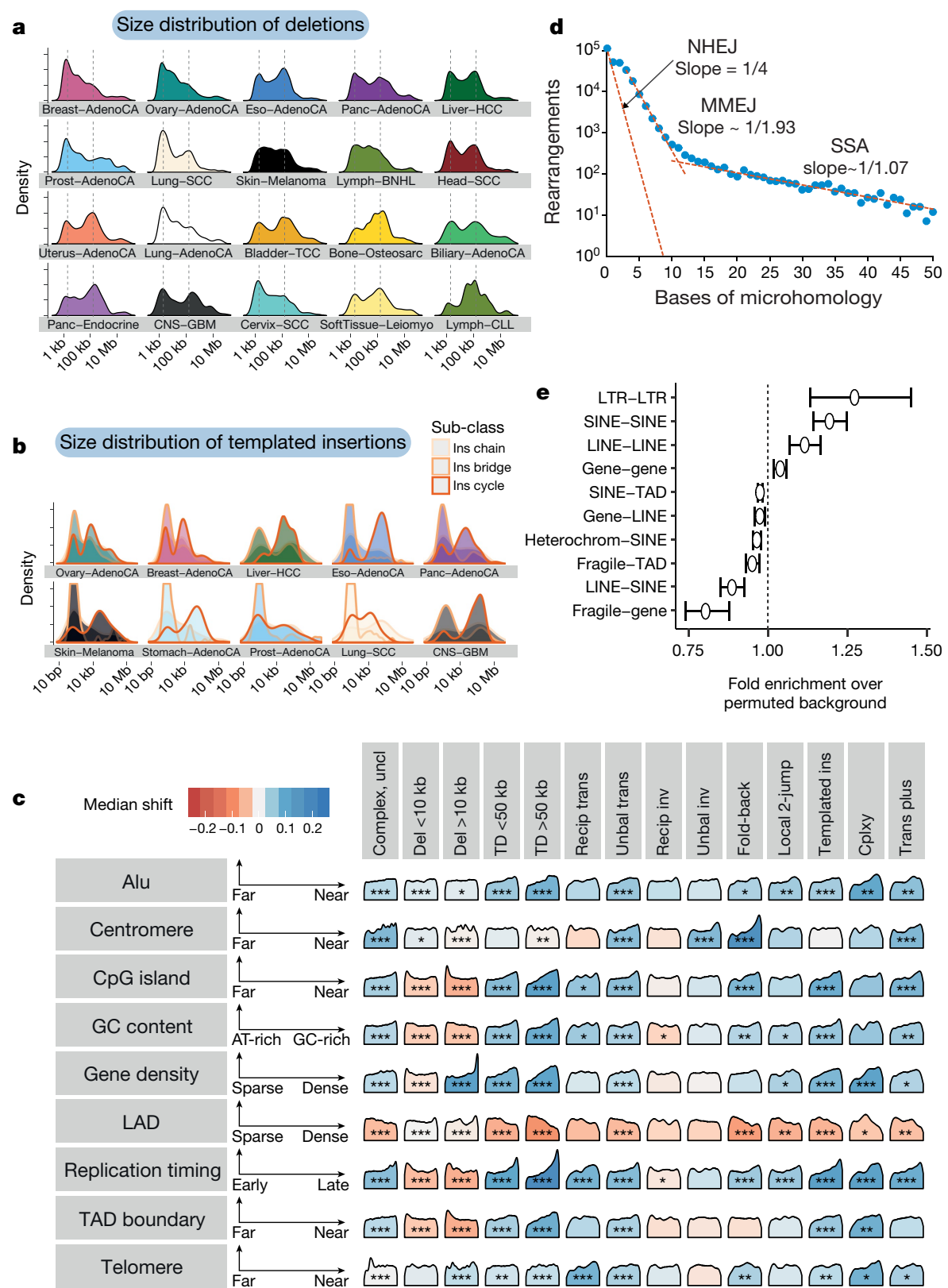

Fig. 5 | Size distribution and genomic properties of classified structural variants. a, Size distribution of deletions per histology group, with tumour types ordered according to total number of events seen. Vertical dashed lines represent the two prominent modes. b. Size distribution of segments of templated insertion per histology group. For each tumour type, the three distributions for cycles, bridges and chains of templated insertions are superimposed. Ins, insertion. c, Associations between a subset of the genomic properties (rows) and classes of structural variant (columns). Each density curve represents the quantile distribution of the genomic property values at observed breakpoints compared to random genome positions. Asterisks indicate a significant departure from uniform quantiles after multiple hypothesis correction on a one-sided Kolmogorov-Smirnov test based on a sample size of 2,559 genomes containing structural variants: $*$ false-discovery rate $<0.01,{ }^{* *}$ false-discovery rate $<0.001,{ }^{* * *}$ false-discovery rate $<10^{-6}$. Cells with significant property associations are shaded by the magnitude of the shift of the median observed quantile above (blue) or below (red) 0.5 . The

range of more-complex patterns. In some cases, the source of the inserted fragment was distal to the major break, and the structural variant could feasibly result from several concurrent DNA breaks in close spatial proximity to the capture of a short DNA fragment during repair (cut-and-paste). In other cases, the origin of the inserted fragment interpretation of each property from left to right is indicated by the axes to the right of the property label. Complex uncl, complex clusters unclassified; cplxy, chromoplexy; del, deletion; inv, inversion; ins, insertion; LAD, laminaassociated domain; recip, reciprocal; TAD, topologically associated domain; TD, tandem duplication; trans, translocation; unbal, unbalanced.

d, Rearrangement counts as a function of bases of junction microhomology, fit to three linear functions consistent with different formation mechanisms. NHEJ, non-homologous end joining; MMEJ, microhomology-mediated end joining; SSA, single-strand annealing. e, Enrichment or depletion of breakpoint junctions between regions of the genome with particular annotations, compared with a permuted background that preserves breakpoint positions but swaps breakpoint partners. Centre points are the mean fold change over the permuted background; error bars represent three s.d. Analysis is based on a sample size of 2,559 genomes containing structural variants. LTR, long terminal repeat; SINE, short interspersed nuclear element; LINE, long interspersed nuclear element; heterochrom, heterochromatin.

was proximal to the major break and associated with a gain in copy number. This pattern is difficult to explain by a cut-and-paste mechanism, because the copy-number gain implies the inserted segment was a duplicate of the original template rather than a separated fragment redistributed from its original locus. Instead, a copy-and-paste 
mechanism may be the more parsimonious explanation for these events.

A comparison of local footprints linked together through distant rearrangements revealed a strong connectivity of footprints with the same or similar structure, often enriched tenfold or more than expected by chance (see 'Footprint connectivity analysis' in Supplementary Results). The reasons for this are unclear, but it may reflect innate structural symmetry introduced through the generation or the resolution of rearrangements, or through the repeated action of a mechanism that imparts consistent structural motifs.

\section{Copy-and-paste patterns of clusters}

The diverse patterns of $2-10$ clustered structural variants (Figs. 3, 4) share important morphological features: (1) genomic configurations that can be phased to a single derivative chromosome; (2) low-level gains in copy number, especially duplications and triplications; (3) a high frequency of inverted rearrangements in addition to noninverted rearrangements; (4) occurrence on a chromosome background with similar average copy number to the tumour overall; and (5) tight proximity of breakpoints within the local footprint (typically $<1 \mathrm{Mb}$ ).

Using our in silico library of genomic configurations, we could define all possible routes by which sequential structural variants could generate these structures through the classically defined repertoire of deletion, tandem duplication, inversion and translocation (Supplementary Fig. 4). These routes typically would require implausible machinations of chromosomes (Supplementary Results). In particular, the high prevalence of inverted breakpoint junctions and local copy-number gains is difficult to recreate using sequential simple rearrangements. Simple inversion events are uncommon in cancers (Fig. 1d) and they tend not to generate copy-number gains, except through breakage-fusion-bridge cycles: these latter also cause terminal deletion ${ }^{2}$, which are not seen in the events discussed here.

If these events cannot be satisfactorily explained by sequential simple rearrangements, another possible explanation is a complex cut-and-paste mechanism such as chromothripsis, chromoplexy or repeated breakage-fusion-bridge cycles. However, the patterns of the $2-10$ clustered structural variants do not fit with these processes either (Supplementary Results). Although chromothripsis with copynumber gain has previously been described ${ }^{3,11,19,22}$, the resulting copy number and rearrangement patterns have different properties to those we observed. Chromoplexy, in which chromosome breaks lead to a balanced interchange at multiple breakpoint junctions ${ }^{5,20}$, typically generates unphased solutions. Repeated breakage-fusion-bridge cycles tend to cause high-level copy-number gains associated with inverted, fold-back rearrangements ${ }^{1,2}$, unlike the structures reported here.

Instead, we believe that many of these locally complex clusters of structural variants with low-level copy-number gains are generated in a single event by a copy-and-paste process. That is, the copying of genomic templates is an intrinsic aspect of the structural variation process in these events, with the extra copies being inserted in the resulting derivative chromosome. If the genomic templates all originate locally, we would observe local $n$-jumps (such as in Fig. $3 a$, b) with a tight clustering of breakpoints, phased solutions, frequent copy-number gains and a mix of inverted and noninverted breakpoint junctions. If the original templates for the copied segments derive from across the genome, chains, cycles and bridges of templated insertions would arise (Fig. 2).

\section{Genomic properties of structural variants}

The size of tandem duplications and deletions followed complex-often multimodal-distributions across tumour types (Fig. 5a, Extended Data Fig. 6a). However, as previously reported ${ }^{6,29}$, individual patients tend to have a simpler-usually unimodal-distribution of deletions or tandem duplications (Extended Data Fig. 6b), which implies that the complexity seen in a given tumour type results from combining samples with different profiles. The sizes of individual fragments in templated insertion events were also distinctly multimodal, with varying peak heights across tumour types (Fig. 5b). When correlating template sizes within a given event, two patterns emerged: one in which template sizes were closely correlated with one another, and one in which a small $(<1 \mathrm{~kb})$ template was linked with one of any size (Extended Data Fig. 7a, b). Likewise, the sizes of segments within a given local two-jump event showed moderately strong correlations with one another (Extended Data Fig. 7c).

A number of genomic properties (such as replication timing, transcriptional activity and chromatin state) influence the density of point mutations $\mathrm{s}^{30,31}$ and copy-number alterations $\mathrm{s}^{32}$, but how this relates to individual classes of structural variant is unclear. From the literature, we compiled a library of the genome-wide distribution of 38 features including replication timing, GC content, repeat density, gene density and distance to G-quadruplex motifs, among others. Replication timing had the strongest association with the occurrence of structural variants; deletions are enriched in late-replicating regions, and tandem duplications and unbalanced translocations occur preferentially in early-replicating regions (Fig. 5c, Extended Data Fig. 8). For individual patients with high numbers of deletions or tandem duplications, we observed notable heterogeneity in the distribution of these structural variants according to replication timing: some had events that occurred predominantly in late-replicating regions, others had events that occurred exclusively in early-replicating regions, and in others events were distributed more evenly (Supplementary Fig. 5). Regions of active chromatin and increased gene density correlated positively with the rate of rearrangement.

A structural variant requires DNA repair pathways to join two sequences together, and several repair mechanisms are available to somatic cells. Some require sequence homology between the two ends, and others can operate to join non-homologous sequences. As previously reported ${ }^{2,25,33}$, we find across the PCAWG data that many structural variants do not have sequence homology at the breakpoint junction (Fig. 5d) and therefore arise through non-homologous end joining. Nonetheless, a sizable fraction of structural variants has more microhomology than expected by chance, with an apparently bimodal distribution of microhomology lengths. One set of structural variants has 2-7 bp of microhomology, probably generated by microhomology-mediated end joining, and a second set of structural variants has 10-30 bp of microhomology, probably generated through single-strand annealing or other forms of homologous recombination (including microhomology-mediated break-induced replication). Repetitive sequences in the genome, such as short and long interspersed nuclear elements, are the likely substrate of such structural variants, and we find enrichment for structural variants joining such elements (Fig. 5e, Supplementary Fig. 6).

\section{Signatures of structural variation}

The heterogeneous spectrum of point mutations across cancers can be reconstructed from the differential action of a relatively limited repertoire of mutational processes, each with a characteristic signature $^{34}$. The differences across patients in the size distribution of tandem duplication and deletion-together with the widely varying frequency and patterns of structural variant across tumour types and genome topology-suggested that we could similarly learn such correlations across individual classes of structural variant.

We divided the set of structural variants of each patient into mutually exclusive categories. We split the most frequent classes of simple structural variant (deletions and tandem duplications) into 11 categories according to size, replication timing and occurrence at fragile sites. Other configurations of structural variants and copy-number changes seen more than 50 times in the cohort were included as further 

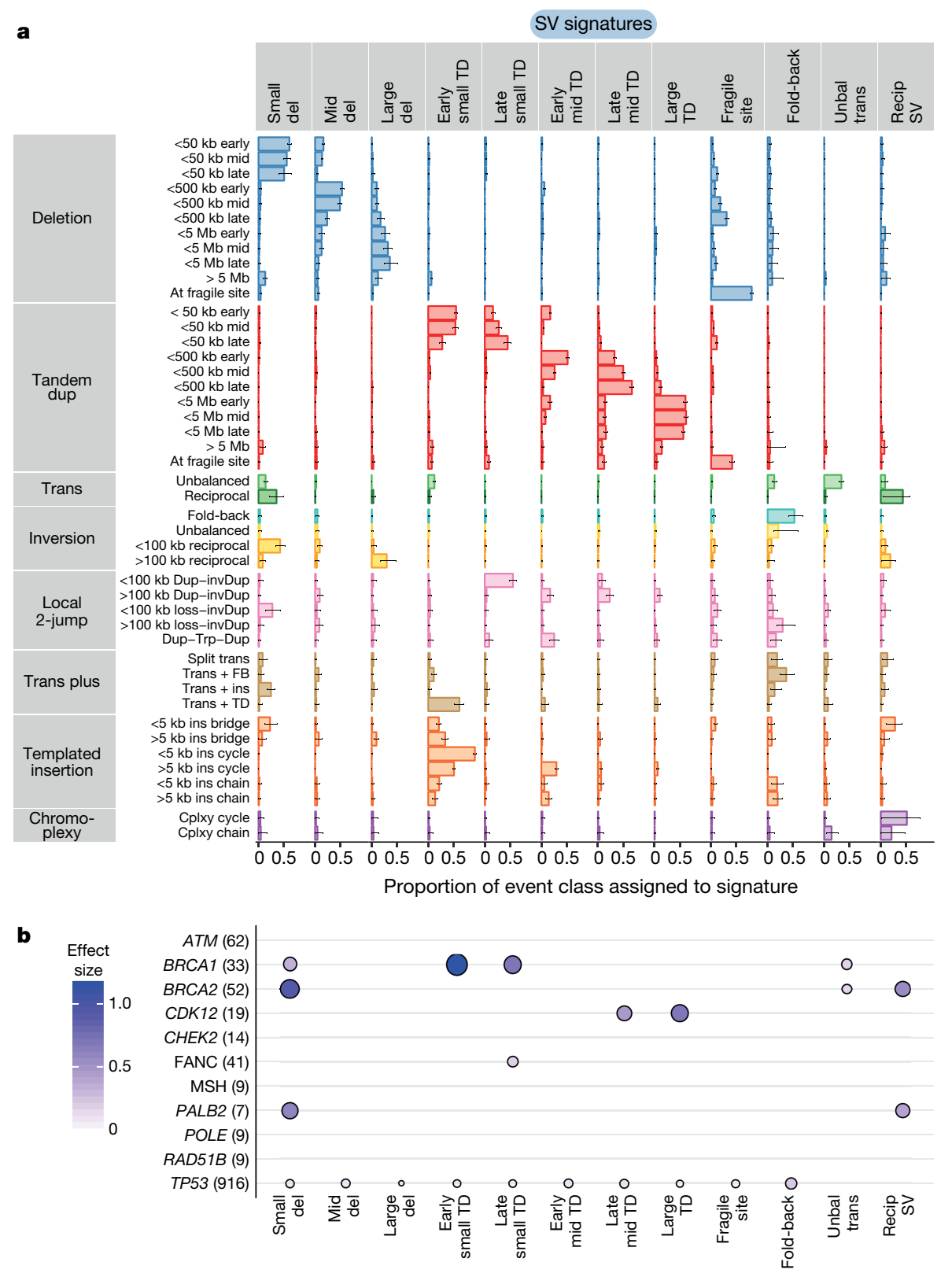

Fig. 6 | Structural-variant signatures in human cancers. a, The 12 most distinctive structural-variant signatures extracted by the Bayesian hierarchical Dirichlet process algorithm, run on a sample size of 2,559 genomes containing structural variants. Here the lengths of the bars represent the estimated proportion of each event class assigned to each signature (rows sum to one); the black line segments represent the $95 \%$ posterior interval for bar length from the Markov chain. FB, fold-back; mid, mid-sized. $\mathbf{b}$, Association of pathogenic mutations (germline and somatic combined) in key DNA repair genes with structural-variant signatures. The sample size of patients who have

categories, including cycles, chains and bridges of templated insertions (also split by size), local $n$-jumps and local-distant clusters.

We applied two methods for signature discovery, which yielded comparable results. We identified 16 structural-variant signatures: the 12 most prevalent of these signatures are shown in Fig. 6a. Signature extraction on the cohort randomly split into two halves identified ten highly correlated signatures (Supplementary Fig. 7), which closely matched the signatures called in the full cohort despite the lower power. Three signatures of deletions emerged, split by size: the signature of small $(<50-\mathrm{kb})$ deletions included small reciprocal inversions and the signature of large $(>500-\mathrm{kb})$ deletions included large reciprocal inversions. This implies that the frequencies of deletions and reciprocal pathogenic variants in the specific genes assessed is shown in brackets after each gene label ( $y$ axis). Hypothesis tests and effect sizes for each gene are derived from linear models for signature intensity after correction for histology. Significant associations from two-sided tests with correction for multiple hypothesis testing are shown. The colour and size of the points represent the estimated effect sizes. MSH refers to $\mathrm{MSH} 2, \mathrm{MSH} 3, \mathrm{MSH} 4$ and $M S H 6$, genes in the mismatch repair pathway; FANC refers to genes associated with Fanconi anaemia, namely FANCA, FANCC, FANCD2, FANCE, FANCF, FANCG, FANCI, FANCL and FANCM.

inversions are correlated across the cohort, and both follow similar size distributions within an individual patient.

We identified five signatures of tandem duplications, split by size and replication timing. Cycles, bridges and chains of templated insertions were particularly prominent in signatures of early-replicating tandem duplications, whereas local two-jump structures were more closely associated with late-replicating tandem duplications. All of these patterns exemplify the copy-and-paste concept, in which extra copies of genomic templates are produced and inserted as an integral feature of the structural-variant process.

Another signature was characterized by deletions and tandem duplications at chromosomal fragile sites ${ }^{35}$. Tandem duplications were more 
prominent at the edges of the fragile site, and deletions were concentrated in the centre (Extended Data Fig. 9a, b). The size range of fragile site deletions peaked at around $100 \mathrm{~kb}$, similar to the larger deletion signature, whereas the rarer fragile-site tandem duplications showed no strong size peak (Extended Data Fig. 9c). Sites of fragility varied extensively across tumour types (Extended Data Fig. 9d).

Unbalanced translocations comprised their own signature, which suggests that they derive from a distinct rearrangement process in cancer genomes. A further signature comprised both the fold-back inversions that are a hallmark of breakage-fusion-bridge cycles and similar structures such as translocations adjacent to fold-back inversions. Finally, there was a signature of balanced rearrangements, including reciprocal translocations and chromoplexy clusters ${ }^{5}$. This signature probably arises from several double-stranded DNA breaks (potentially occurring in interphase), in which both sides of the break are incorrectly repaired through ligation to other, simultaneously broken regions of the genome.

\section{DNA repair genes and tumour type}

We grouped annotations of pathogenic germline variants and somatic driver mutations in DNA-repair genes across the cohort ${ }^{8}$, correlating their presence with activity of the structural-variant signatures (Fig. 6b). As previously described for breast and ovarian cancers ${ }^{6,29}$, BRCA1 mutations are significantly associated with small tandem duplication signatures, the mechanistic basis of which is increasingly well understood $^{10}$. As previously described ${ }^{6,36}, C D K 12$ variants predicted signatures of mid-sized-to-large tandem duplications. BRCA2 variants correlated with small deletions, as expected from previous work ${ }^{29}$, and also with the reciprocal structural-variant signature that includes chromoplexy. PALB2 variants showed the same correlations with signatures of small deletions and reciprocal structural variants as does BRCA2: PALB2 colocalizes with, stabilizes and assists BRCA2 during homologous recombination ${ }^{37}$, so we might have predicted that inactivation of either gene would lead to a similar structural-variant signature. These associations between driver mutations and structural-variant signatures were consistently evident across many types of tumour (Extended Data Fig. 10).

The structural-variant signatures showed considerable heterogeneity in their activity across tumour types and among patients within a given tumour type (Supplementary Fig. 8). Tumours of the gastrointestinal tract-including colorectal and oesophageal adenocarcinomas-showed high rates of the fragile-site signature. Prostate cancer was notable for the prevalence of the chromoplexy signature, as previously reported ${ }^{5,20}$, and squamous cell carcinomas of the lung were characterized by the fold-back inversion signature.

We assessed how classes of structural variant altered known cancer genes (Supplementary Table 1). Some cancer genes acquire oncogenic potential only with specific structural events, such as fusion genes or enhancer hijacking. Not surprisingly, these genes typically showed little variability in which classes of structural variant could generate such events (Extended Data Fig. 11a-c)-although there were exceptions. The TMPRSS2-ERG fusion gene of prostate cancer, for example, was generated by a range of processes (including simple deletions, chromoplexy and chromothripsis), all of which are prevalent signatures in this tumour type (Extended Data Fig. 11d-f).

Tumour-suppressor genes and recurrently amplified genes showed more variability in which types of structural variant were observed, and these were shaped by signatures active in the relevant tumour types. For example, the tumour-suppressor genes, PTEN and RAD51B, which are commonly inactivated in breast and ovarian cancers, were often targeted by tandem duplications generating out-of-frame exon duplications (Extended Data Fig. 12a, b). By contrast, deletions were the predominant events that inactivated SMAD4 and $C D K N 2 A$, in keeping with their prevalence in cancers of the gastrointestinal tract
(Extended Data Fig. 12c, d).MYC, one of the most commonly amplified genes across all types of cancer, showed considerable diversity in the mechanisms of its rearrangement: nested tandem duplications in breast cancer, translocations or chromoplexy with /GH in lymphoma, as well as chromothripsis, cycles of templated insertions, local $n$-jumps and local-distant clusters in other types of tumour (Extended Data Fig.13).

\section{Discussion}

We have described the patterns and signatures of structural variation in a large cohort of uniformly analysed cancer genomes. A major grouping of patterns in structural variants that emerges from our study is one in which extra copies of genomic templates are inserted during the rearrangement process. This includes simple events such as tandem duplications, as well as a range of more-complex events with duplications and triplications that are rearranged locally as well as inserted distantly. Our signature analysis grouped a large proportion of these more-complex events together with tandem duplications, which suggests that they represent a continuum of processes that share underlying properties. A replication-based mechanism has previously been proposed to explain local two-jumps ${ }^{4,23,24}$, in which stalled replication forks or other DNA lesions cause the DNA polymerase to switch templates and continue replication in a new location. Studies in experimental models are now revealing that a wide range of mechanisms and DNA lesions can result in templated insertions: these mechanisms include tandem duplications in $B R C A 1$ deficiency ${ }^{10}$, translocations with templated insertions caused by dysregulated strand invasion ${ }^{38}$ and distant templated insertions in the absence of replication helicases ${ }^{39}$.

Genomic instability in cancer is not a single phenomenon. Instead, many different mutational processes can act to restructure the genome and, in doing so, generate a notably flexible array of possible structures. Any given tumour draws on a subset of the available processes, shaped by the cell of origin, germline predisposition and other, unknown, factors: selection then does the rest, promoting the clone that has chanced on the structure that increases its potential for self-determination.

\section{Online content}

Any methods, additional references, Nature Research reporting summaries, source data, extended data, supplementary information, acknowledgements, peer review information; details of author contributions and competing interests; and statements of data and code availability are available at https://doi.org/10.1038/s41586-019-1913-9.

1. Bignell, G. R. et al. Architectures of somatic genomic rearrangement in human cancer amplicons at sequence-level resolution. Genome Res. 17, 1296-1303 (2007).

2. Campbell, P. J. et al. The patterns and dynamics of genomic instability in metastatic pancreatic cancer. Nature 467, 1109-1113 (2010).

3. Stephens, P. J. et al. Massive genomic rearrangement acquired in a single catastrophic event during cancer development. Cell 144, 27-40 (2011).

4. Lee, J. A., Carvalho, C. M. \& Lupski, J. R. A DNA replication mechanism for generating nonrecurrent rearrangements associated with genomic disorders. Cell 131, 1235-1247 (2007).

5. Baca, S. C. et al. Punctuated evolution of prostate cancer genomes. Cell 153, 666-677 (2013).

6. Menghi, F. et al. The tandem duplicator phenotype is a prevalent genome-wide cancer configuration driven by distinct gene mutations. Cancer Cell 34, 197-210 (2018).

7. Liu, P. et al. An organismal CNV mutator phenotype restricted to early human development. Cell 168, 830-842 (2017).

8. The ICGC/TCGA Pan-Cancer Analysis of Whole Genomes Consortium. Pan-cancer analysis of whole genomes. Nature https://doi.org/10.1038/s41586-020-1969-6 (2020).

9. Zhang, C.-Z. et al. Chromothripsis from DNA damage in micronuclei. Nature 522, 179-184 (2015).

10. Willis, N. A. et al. Mechanism of tandem duplication formation in BRCA1-mutant cells. Nature 551, 590-595 (2017).

11. Maciejowski, J., Li, Y., Bosco, N., Campbell, P. J. \& de Lange, T. Chromothripsis and kataegis induced by telomere crisis. Cell 163, 1641-1654 (2015).

12. Ly, P. et al. Chromosome segregation errors generate a diverse spectrum of simple and complex genomic rearrangements. Nat. Genet. 51, 705-715 (2019).

13. Ghezraoui, H. et al. Chromosomal translocations in human cells are generated by canonical nonhomologous end-joining. Mol. Cell 55, 829-842 (2014). 
14. Rheinbay, E. et al. Analyses of non-coding somatic drivers in 2,658 cancer whole genomes. Nature https://doi.org/10.1038/s41586-020-1965-x (2020).

15. PCAWG Transcriptome Core Group et al. Genomic basis for RNA alterations in cancer. Nature https://doi.org/10.1038/s41586-020-1970-0 (2020).

16. Akdemir, K. C. et al. Disruption of chromatin folding domains by somatic genomic rearrangements in human cancer. Nat. Genet. https://doi.org/10.1038/s41588-019-0564-y (2020).

17. Rodriguez-Martin, B. et al. Pan-cancer analysis of whole genomes identifies driver rearrangements promoted by LINE-1 retrotransposition. Nat. Genet. https://doi.org/ 10.1038/s41588-019-0562-0 (2020)

18. Cortes-Ciriano, I. et al. Comprehensive analysis of chromothripsis in 2,658 human cancers using whole-genome sequencing. Nat. Genet. https://doi.org/10.1038/s41588019-0576-7 (2020).

19. Li, Y. et al. Constitutional and somatic rearrangement of chromosome 21 in acute lymphoblastic leukaemia. Nature 508, 98-102 (2014)

20. Berger, M. F. et al. The genomic complexity of primary human prostate cancer. Nature 470, 214-220 (2011).

21. Crasta, K. et al. DNA breaks and chromosome pulverization from errors in mitosis. Nature 482, 53-58 (2012)

22. Rausch, T. et al. Genome sequencing of pediatric medulloblastoma links catastrophic DNA rearrangements with TP53 mutations. Cell 148, 59-71 (2012).

23. Hastings, P. J., Ira, G. \& Lupski, J. R. A microhomology-mediated break-induced replication model for the origin of human copy number variation. PLoS Genet. 5, e1000327 (2009).

24. Carvalho, C. M. B. et al. Inverted genomic segments and complex triplication rearrangements are mediated by inverted repeats in the human genome. Nat. Genet. 43, 1074-1081 (2011)

25. Campbell, P. J. et al. Identification of somatically acquired rearrangements in cancer using genome-wide massively parallel paired-end sequencing. Nat. Genet. 40, 722-729 (2008).

26. Rausch, T. et al. DELLY: structural variant discovery by integrated paired-end and splitread analysis. Bioinformatics 28, i333-i339 (2012).

27. Wala, J. A. et al. SvABA: genome-wide detection of structural variants and indels by local assembly. Genome Res. 28, 581-591 (2018).

28. Totoki, Y. et al. Trans-ancestry mutational landscape of hepatocellular carcinoma genomes. Nat. Genet. 46, 1267-1273 (2014).

29. Nik-Zainal, S. et al. Landscape of somatic mutations in 560 breast cancer whole-genome sequences. Nature 534, 47-54 (2016)

30. Supek, F. \& Lehner, B. Differential DNA mismatch repair underlies mutation rate variation across the human genome. Nature 521, 81-84 (2015).

31. Schuster-Böckler, B. \& Lehner, B. Chromatin organization is a major influence on regional mutation rates in human cancer cells. Nature 488, 504-507 (2012).

32. De, S. \& Michor, F. DNA replication timing and long-range DNA interactions predict mutational landscapes of cancer genomes. Nat. Biotechnol. 29, 1103-1108 (2011).

33. Yang, L. et al. Diverse mechanisms of somatic structural variations in human cancer genomes. Cell 153, 919-929 (2013).

34. Alexandrov, L. B. et al. The repertoire of mutational signatures in human cancer. Nature https://doi.org/10.1038/s41586-020-1943-3 (2020).

35. Lukusa, T. \& Fryns, J. P. Human chromosome fragility. Biochim. Biophys. Acta 1779, 3-16 (2008).

36. Popova, T. et al. Ovarian cancers harboring inactivating mutations in CDK12 display a distinct genomic instability pattern characterized by large tandem duplications. Cancer Res. 76, 1882-1891 (2016).

37. Xia, B. et al. Control of BRCA2 cellular and clinical functions by a nuclear partner, PALB2. Mol. Cell 22, 719-729 (2006).

38. Piazza, A., Wright, W. D. \& Heyer, W. D. Multi-invasions are recombination byproducts that induce chromosomal rearrangements. Cell 170, 760-773 (2017).

39. Yu, Y. et al. Dna2 nuclease deficiency results in large and complex DNA insertions at chromosomal breaks. Nature 564, 287-290 (2018).

Publisher's note Springer Nature remains neutral with regard to jurisdictional claims in published maps and institutional affiliations.

Open Access This article is licensed under a Creative Commons Attribution 4.0 International License, which permits use, sharing, adaptation, distribution and reproduction in any medium or format, as long as you give appropriate credit to the
original author(s) and the source, provide a link to the Creative Commons license, and indicate if changes were made. The images or other third party material in this article are included in the article's Creative Commons license, unless indicated otherwise in a credit line to the material. If material is not included in the article's Creative Commons license and your intended use is not permitted by statutory regulation or exceeds the permitted use, you will need to obtain permission directly from the copyright holder. To view a copy of this license visit http://creativecommons.org/licenses/by/4.0/.

(c) The Author(s) 2020, corrected publication 2023

PCAWG Structural Variation Working Group

Kadir C. Akdemir ${ }^{14}$, Eva G. Alvarez ${ }^{15,16,17}$, Adrian Baez-Ortega ${ }^{18}$, Rameen Beroukhim ${ }^{3,4,5}$, Paul C. Boutros $^{19,20,21,22}$, David D. L. Bowtell ${ }^{23,24}$, Benedikt Brors ${ }^{25,26,27}$, Kathleen H. Burns ${ }^{28}$, Peter J. Campbell $^{1,12}$, Kin Chan ${ }^{29}$, Ken Chen $^{14}$, Isidro Cortés-Ciriano ${ }^{30,31,32}$, Ana Dueso-Barroso ${ }^{33}$, Andrew J. Dunford ${ }^{3}$, Paul A. Edwards ${ }^{34,35}$, Xavier Estivill ${ }^{36,37}$, Dariush Etemadmoghadam ${ }^{23}$ Lars Feuerbach $^{26}$, J. Lynn Fink ${ }^{33,38}$, Milana Frenkel-Morgenstern ${ }^{39}$, Dale W. Garsed ${ }^{23}$, Mark Gerstein $^{40,41,42}$, Dmitry A. Gordenin ${ }^{43}$, David Haan ${ }^{44}$, James E. Haber ${ }^{8}$, Julian M. Hess ${ }^{3,45}$, Barbara Hutter ${ }^{25,27,46}$, Marcin Imielinski ${ }^{6,9}$, David T. W. Jones ${ }^{47,48}$, Young Seok Ju',49, Marat D.
Kazanov $^{50,51,52}$, Leszek J. Klimczak ${ }^{53}$, Youngil Koh ${ }^{54,55}$, Jan O. Korbel ${ }^{7}$, Kiran Kumar ${ }^{3}$, Eunjung Alice Lee ${ }^{56,57}$, Jake June-Koo Lee ${ }^{31,32}$, Yilong Li ${ }^{1,2}$, Andy G. Lynch ${ }^{34,35,58}$, Geoff Macintyre ${ }^{34,35}$, Florian Markowetz ${ }^{34,35}$, Iñigo Martincorena', Alexander Martinez-Fundichely ${ }^{59,60,61}$, Matthew Meyerson $^{3,4,62}$, Satoru Miyano ${ }^{63}{ }^{\text {, Hidewaki Nakagawa }}{ }^{64}$, Fabio C. P. Navarro ${ }^{65}$, Stephan Ossowski ${ }^{66,67,68}$, Peter J. Park ${ }^{31,32}$, John V. Pearson ${ }^{69,70}$, Montserrat Puiggròs ${ }^{33}$, Karsten Rippe ${ }^{71}$, Nicola D. Roberts', Steven A. Roberts ${ }^{72}$, Bernardo Rodriguez-Martin ${ }^{15,16,17}$, Steven E. Schumacher $^{3,4,5}$, Ralph Scully ${ }^{73}$, Mark Shackleton ${ }^{23,24}$, Nikos Sidiropoulos ${ }^{11}$, Lina Sieverling ${ }^{26,74}$, Chip Stewart ${ }^{3}$, David Torrents ${ }^{33,75}$, Jose M. C. Tubio ${ }^{15,16,17}$, Izar Villasante ${ }^{33}$, Nicola Waddel ${ }^{69,70}$, Jeremiah A. Wala ${ }^{3,4,5}$, Joachim Weischenfeldt ${ }^{11}$, Lixing Yang ${ }^{76}$, Xiaotong Yao $^{9,77}$, Sung-Soo Yoon ${ }^{55}$, Jorge Zamora ${ }^{1,15,16,17}$ \& Cheng-Zhong Zhang ${ }^{3,4,62}$

${ }^{14}$ University of Texas MD Anderson Cancer Center, Houston, TX, USA. ${ }^{15}$ Department of Zoology, Genetics and Physical Anthropology, University of Santiago de Compostela, Santiago de Compostela, Spain. ${ }^{16}$ Centre for Research in Molecular Medicine and Chronic Diseases (CIMUS), University of Santiago de Compostela, Santiago de Compostela, Spain ${ }^{17}$ The Biomedical Research Centre (CINBIO), University of Vigo, Vigo, Spain. ${ }^{18}$ Transmissible Cancer Group, Department of Veterinary Medicine, University of Cambridge, Cambridge, UK. ${ }^{19}$ Computational Biology Program, Ontario Institute for Cancer Research, Toronto, Ontario, Canada. ${ }^{20}$ Department of Medical Biophysics, University of Toronto, Toronto, Ontario, Canada. ${ }^{21}$ Department of Pharmacology, University of Toronto, Toronto, Ontario, Canada. ${ }^{22}$ University of California Los Angeles, Los Angeles, CA, USA. ${ }^{23}$ Peter MacCallum Cancer Centre, Melbourne, Victoria, Australia. ${ }^{24}$ Sir Peter MacCallum Department of Oncology, University of Melbourne, Melbourne, Victoria, Australia. ${ }^{25}$ National Center for Tumor Diseases (NCT) Heidelberg, Heidelberg, Germany. ${ }^{26}$ Division of Applied Bioinformatics, German Cancer Research Center (DKFZ), Heidelberg, Germany. ${ }^{27}$ German Cancer Genome Consortium (DKTK), Heidelberg, Germany. ${ }^{28}$ Johns Hopkins School of Medicine, Baltimore, MD, USA. ${ }^{29}$ Faculty of Medicine, Department of Biochemistry, Microbiology and Immunology, University of Ottawa, Ottawa, Ontario, Canada. ${ }^{30}$ Centre for Molecular Science Informatics, Department of Chemistry, University of Cambridge, Cambridge, UK. ${ }^{31}$ Department of Biomedical Informatics, Harvard Medical School, Boston, MA, USA. ${ }^{32}$ Ludwig Center, Harvard Medical School, Boston, MA, USA. ${ }^{33}$ Barcelona Supercomputing Center (BSC), Barcelona, Spain. ${ }^{34}$ Cancer Research UK Cambridge Institute, University of Cambridge, Cambridge, UK. ${ }^{35}$ University of Cambridge, Cambridge, UK. ${ }^{36}$ Sidra Medicine, Doha, Qatar. ${ }^{37}$ Barcelona Supercomputing Center (BSC), Barcelona, Spain. ${ }^{38}$ Queensland Centre for Medical Genomics, Institute for Molecular Bioscience, The University of Queensland, Brisbane, Queensland, Australia. ${ }^{39}$ The Azrieli Faculty of Medicine, Bar-Ilan University, Safed, Israel. ${ }^{40}$ Department of Computer Science, Princeton University, Princeton, NJ, USA. ${ }^{41}$ Department of Computer Science, Yale University, New Haven, CT, USA. ${ }^{42}$ Program in Computational Biology and Bioinformatics, Yale University, New Haven, CT, USA. ${ }^{43}$ Genome Integrity and Structural Biology Laboratory, National Institute of Environmental Health Sciences (NIEHS), Durham, NC, USA. ${ }^{44}$ Biomolecular Engineering Department, University of California, Santa Cruz, Santa Cruz, CA, USA. ${ }^{45}$ Massachusetts General Hospital Center for Cancer Research, Charlestown, MA, USA. ${ }^{46}$ Heidelberg Center for Personalized Oncology (DKFZ-HIPO), German Cancer Research Center (DKFZ), Heidelberg, Germany. ${ }^{47}$ Hopp Children's Cancer Center (KiTZ), Heidelberg, Germany. ${ }^{48}$ Pediatric Glioma Research Group, German Cancer Research Center (DKFZ), Heidelberg, Germany. ${ }^{49}$ Korea Advanced Institute of Science and Technology, Daejeon, South Korea. ${ }^{50}$ Skolkovo Institute of Science and Technology, Moscow, Russia. ${ }^{51} \mathrm{~A}$. A. Kharkevich Institute of Information Transmission Problems, Moscow, Russia. ${ }^{52}$ Dmitry Rogachev National Research Center of Pediatric Hematology, Oncology and Immunology, Moscow, Russia. ${ }^{53}$ Integrative Bioinformatics Support Group, National Institute of Environmental Health Sciences (NIEHS), Durham, NC, USA. ${ }^{54}$ Center For Medical Innovation, Seoul National University Hospital, Seoul, South Korea. ${ }^{55}$ Department of Internal Medicine, Seoul National University Hospital, Seoul, South Korea. ${ }^{56}$ Division of Genetics and Genomics, Harvard Medical School, Boston, MA, USA. ${ }^{57}$ Boston Children's Hospital, Boston, MA, USA. ${ }^{58}$ School of Medicine/School of Mathematics and Statistics, University of St Andrews, St Andrews, UK. ${ }^{59}$ Department of Physiology and Biophysics, Weill Cornell Medicine, New York, NY, USA. ${ }^{60}$ Institute for Computational Biomedicine, Weill Cornell Medicine, New York, NY, USA. ${ }^{61}$ Englander Institute for Precision Medicine, Weill Cornell Medicine, New York, NY, USA. ${ }^{62}$ Dana-Farber Cancer Institute, Boston, MA, USA. ${ }^{63}$ The Institute of Medical Science, The University of Tokyo, Tokyo, Japan. ${ }^{64}$ RIKEN Center for Integrative Medical Sciences, Yokohama, Japan. ${ }^{65}$ Department of Molecular Biophysics and Biochemistry, Yale University, New Haven, CT, USA. ${ }^{66}$ Universitat Pompeu Fabra (UPF), Barcelona, Spain. ${ }^{67}$ Centre for Genomic Regulation (CRG), The Barcelona Institute of Science and Technology, Barcelona, Spain. ${ }^{68}$ Institute of Medical Genetics and Applied Genomics, University of Tübingen, Tübingen, Germany. ${ }^{69}$ Department of Genetics and Computational Biology, QIMR Berghofer Medical Research Institute, Brisbane, Queensland, Australia. ${ }^{70}$ Institute for Molecular Bioscience, University of Queensland, Brisbane, Queensland, Australia. ${ }^{71}$ German Cancer Research Center (DKFZ), Heidelberg, Germany. ${ }^{72}$ School of Molecular Biosciences and Center for Reproductive Biology, Washington State University, Pullman, WA, USA. ${ }^{73}$ Cancer Research Institute, Beth Israel Deaconess Medical Center, Boston, MA, USA. ${ }^{74}$ Faculty of Biosciences, Heidelberg University, Heidelberg, Germany. ${ }^{75}$ Institució Catalana de Recerca i Estudis Avançats (ICREA), Barcelona, Spain. ${ }^{76}$ Ben May Department for Cancer Research, Department of Human Genetics, The University of Chicago, Chicago, IL, USA. ${ }^{77}$ Tri-institutional PhD Program of Computational Biology and Medicine, Weill Cornell Medicine, New York, NY, USA. 


\section{Article}

\section{Methods}

No statistical methods were used to predetermine sample size. The experiments were not randomized and investigators were not blinded to allocation during experiments and outcome assessment.

A detailed description of the methods used in this paper and many additional results are described in Supplementary Information. Here, we summarize the key aspects of the analysis.

\section{Generation of the structural-variant call set}

The final set of structural variants used in this Article was generated by the Technical Working Group of the PCAWG Consortium and is described in the main PCAWG paper ${ }^{8}$. In brief, four variant callers were used to identify somatically acquired structural variants from matched tumour and germline whole genome sequencing data:SvABA (Broad pipeline), DELLY (DKFZ pipeline), BRASS (Sanger pipeline) and dRanger (Broad pipeline). These were merged into a final call set using a graph-based algorithm to identify overlapping breakpoint junctions across algorithms. Detailed visual inspection of structural-variant calls suggested that a simple approach of accepting all structural-variant calls made by two or more of the four algorithms gave the best trade-off between sensitivity and specificity.

\section{Structural-variant clustering and annotation}

To identify clusters of structural variants, we developed a method for grouping structural variants into clusters and footprints to allow structural and mechanistic inferences to be made systematically. In parallel, we processed the somatic copy-number data and merged it with structural-variant junctions to enable us produce rearrangement patterns from the generated structural-variant clusters and footprints. We produced normalized representations of structuralvariant cluster patterns, which enable us to tabulate the number of different cluster and footprint patterns and analyse their features. Finally, we performed manual and simulation-assisted interpretation of the recurrently observed cluster and footprint patterns. The individual steps of the structural-variant classification pipeline are outlined below and detailed in the subsequent subsections: (1) computing the exact breakpoint coordinates from clipped reads; (2) removing redundant 'segment-bypassing' structural variants; (3) merging rearrangement breakpoints with copy-number data to yield structural-variant breakpoint-demarcated, normalized, absolute copy-number data; (4) clustering individual structural variants into structural-variant clusters and footprints; (5) heuristically refining structural-variant clusters and footprints; (6) filtering artefactual fold-back-type structural variants with insufficient support; (7) determining balanced overlapping breakpoints (this step is to distinguish very short templated insertions from mutually overlapping balanced breakpoints); and (8) computing rearrangement patterns and categories.

\section{Distribution of structural variants across the genome}

We divided the hg19 human reference genome (autosomes and chromosome $\mathrm{X}$ ) into $3,036,315$ pixels of $1 \mathrm{~kb}$, and calculated a suite of metrics per pixel to summarize a variety of genome properties with potential relevance to the distribution of rearrangements, as listed in the Supplementary Information. Properties were matched as closely as possible to the tissue of origin for cancer samples from the PCAWG data. All other genome properties were held fixed across all tissues. To test for associations between structural-variant event classes and the library of genome properties, the genome property metrics were compared between real structural-variant positions (randomly choosing one side of each breakpoint junction to reduce dependence between observations) and one million uniform random positions from the callable genome space. To compare the tissue-specific properties, each random position was assigned a random tissue type, drawing from the observed tissue-type distribution in the structural-variant call set.
For each genome property and each event class, the real observations were pooled amongst the random ones, and then rank-transformed and normalized on a scale from 0 to 1 . Under the null hypothesis of no event-versus-property association, the ranks of the real observations would follow a uniform distribution. We tested this in each case with a Kolmogorov-Smirnov test then applied a Benjamini-Yekutieli correction for false-discovery rate across the entire suite of tests and set the threshold for significance reporting at 0.01 .

\section{Structural-variant-signature analysis}

We used two algorithms for extracting structural-variant signatures. Both used the same input files, comprising a matrix of counts per patient (across all patients) of structural-variant clusters falling into a number of mutually exclusive categories. These categories included the major classes of structural variants, with the more-common events (deletions, tandem duplications and inversions) split by size and/or replication timing. The two algorithms that were used for extracting the signatures were (1) a hierarchical Dirichlet process and (2) nonnegative matrix factorization. Further details on the implementation of these algorithms are available in the Supplementary Information.

\section{Reporting summary}

Further information on research design is available in the Nature Research Reporting Summary linked to this paper.

\section{Data availability}

Somatic and germline variant calls, mutational signatures, subclonal reconstructions, transcript abundance, splice calls and other core data generated by the ICGC/TCGA PCAWG Consortium are described in an accompanying Article ${ }^{8}$ and are available for download at https://dcc. icgc.org/releases/PCAWG. Additional information on accessing the data, including raw read files, can be found at https://docs.icgc.org/ pcawg/data/. In accordance with the data access policies of the ICGC and TCGA projects, most molecular, clinical and specimen data are in an open tier that does not require access approval. To access information that could potentially identify participants, such as germline alleles and the underlying sequencing data, researchers will need to apply to the TCGA data access committee via dbGaP (https://dbgap.ncbi.nlm. nih.gov/aa/wga.cgi?page=login) for access to the TCGA portion of the dataset, and to the ICGC data access compliance office (http://icgc.org/ daco) for the ICGC portion of the dataset. In addition, to access somatic single-nucleotide variants derived from TCGA donors, researchers will also need to obtain dbGaP authorization.

\section{Code availability}

The core computational pipelines used by the PCAWG Consortium for alignment, quality control and variant calling are available to the public at https://dockstore.org/search?search=pcawg under the GNU General Public License v.3.0, which allows for reuse and distribution. These are described in detail in an accompanying Article ${ }^{8}$. The code for grouping structural variants into structural-variant clusters and footprints is available at https://github.com/cancerit/ClusterSV/ (version 1.0). The code for simulating rearrangements can be found at https://github.com/cancerit/SimSvGenomes (version 1.0). The code for sampling from the hierarchical Dirichlet process for identification of mutational signatures is implemented as an $\mathrm{R}$ package at https:// github.com/nicolaroberts/hdp (version 0.1.1).

Acknowledgements This work was supported by the Wellcome Trust, Pediatric Low-Grade Astrocytoma Fund and the Fund for Innovation in Cancer Informatics. P.J.C. is a Wellcome Trust Senior Clinical Fellow (WT08834OMA). We acknowledge the contributions of the many clinical networks across ICGC and TCGA, which provided samples and data to the PCAWG Consortium, and the contributions of the Technical Working Group and the Germline Working Group of the PCAWG Consortium for the collation, realignment and harmonized variant- 
calling of the cancer genomes used in this study. We thank the patients and their families for their participation in the individual ICGC and TCGA projects.

Author contributions Y.L., N.D.R., J.A.W. and O.S. contributed equally to this manuscript, undertaking evaluation and curation of structural-variant calls, merging structural-variant cal sets from four separate algorithms into a final dataset. Y.L. performed the clustering and classification of structural variants, and identified patterns of rearrangement, with assistance from N.D.R. and M.I. N.D.R. performed the analysis of structural-variant signatures with assistance from Y.L. N.D.R., J.A.W. and O.S. analysed the distribution of structural variants across the genome, with input from J.E.H., E.K., K.K. and S.E.S. S.W. and J.O.K. contributed to the analysis of how germline variants influenced signatures of structural variants. J.W., R.B. and P.J.C. jointly oversaw the project, assisted with data interpretation and wrote the paper, with input from all authors.
Competing interests R.B. owns equity in Ampressa Therapeutics; M.M. is the scientific advisory board chair of-and consultant for-OrigiMed, and receives research funding from Bayer and Ono Pharma, and patent royalties from LabCorp.; J.W. is a consultant for Nference Inc.; C.-Z.Z. is a cofounder and equity holder of Pillar Biosciences, a for-profit company specializing in the development of targeted sequencing assays.

Additional information

Supplementary information is available for this paper at https://doi.org/10.1038/s41586-0191913-9.

Correspondence and requests for materials should be addressed to J.W., R.B. or P.J.C

Peer review information Nature thanks Don Conrad, Ben Lehner and the other, anonymous, reviewer(s) for their contribution to the peer review of this work.

Reprints and permissions information is available at http://www.nature.com/reprints. 


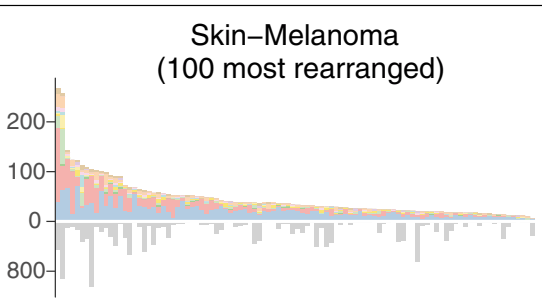

Ovary-AdenoCA

(100 most rearranged)

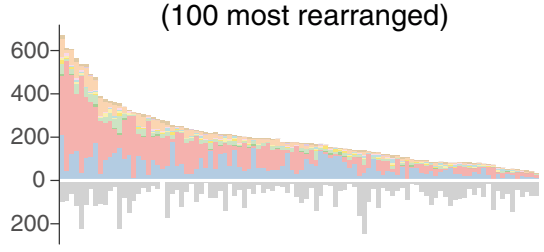

Kidney-RCC

(100 most rearranged)
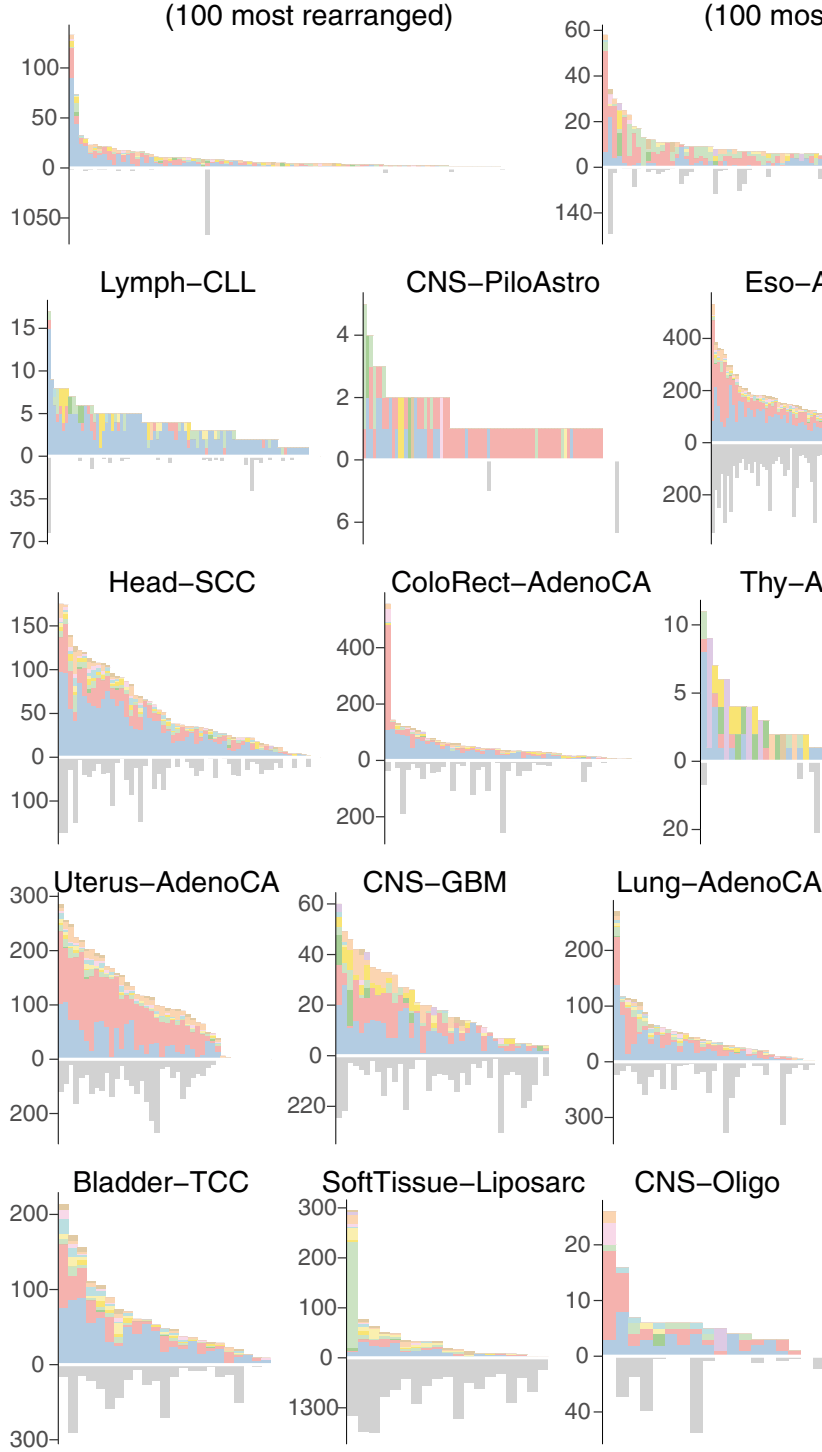

Extended Data Fig. 1 | Per-sample counts of structural-variant breakpoint junctions by histology group. Counts of simple, classified structural variants are shown above the $x$ axis and counts of complex breakpoint junctions below

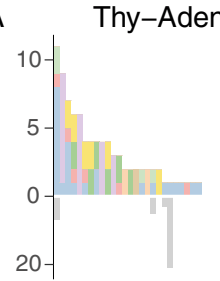

Prost-AdenoCA

(100 most rearranged)

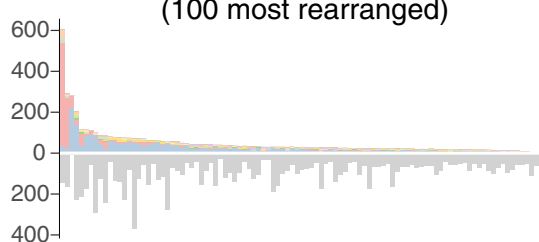

Lymph-BNHL

(100 most rearranged)

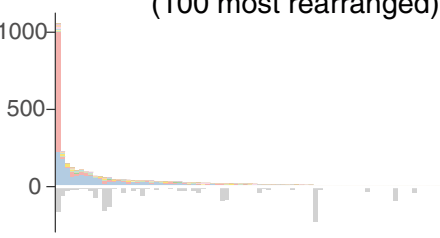

CNS-Medullo

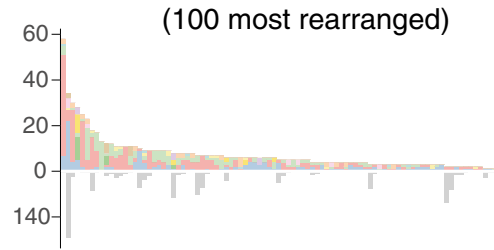

Panc-AdenoCA

(100 most rearranged)

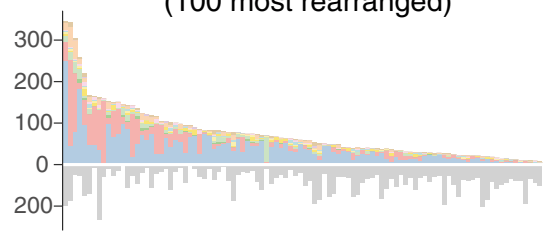

Liver-HCC

(100 most rearranged)

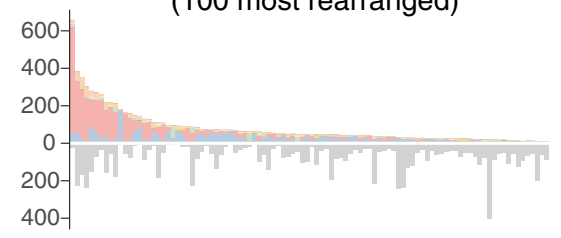

Breast-AdenoCA

(100 most rearranged)

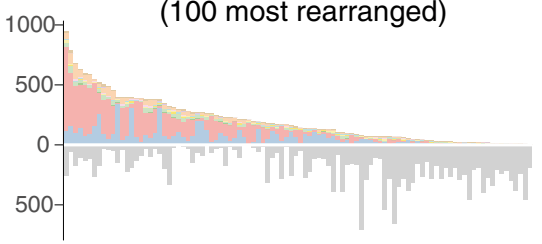

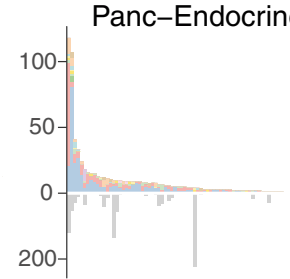

Lung-SCC

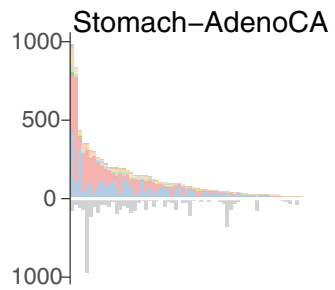

\begin{abstract}
CA
\end{abstract}
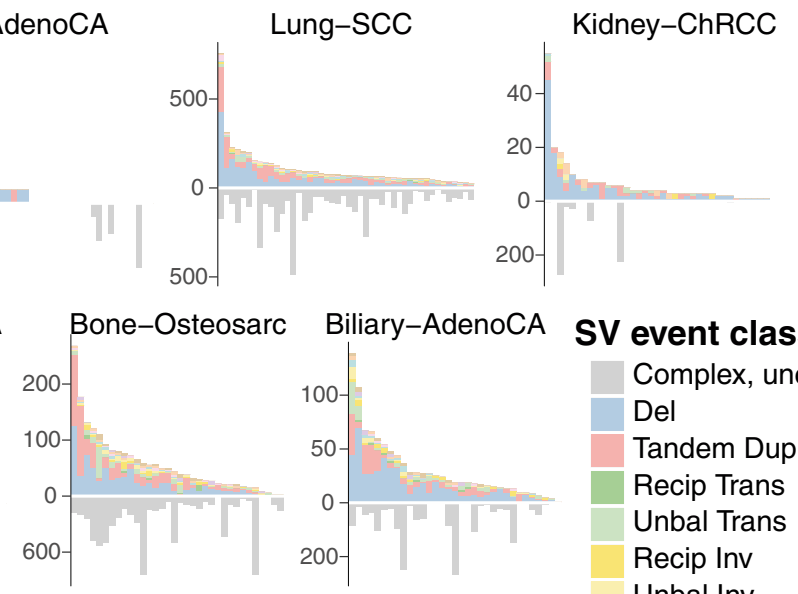

SV event class

Complex, uncl Del Tandem Dup Recip Trans Unbal Trans Recip Inv Unbal Inv
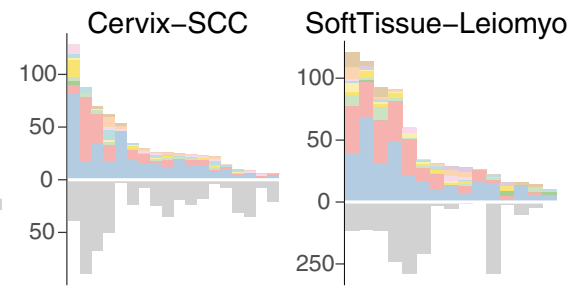

Foldback Loc 2-Jump Templ Ins Cplxy Trans Plus the $x$ axis. Patients within each tumour type are ranked by frequency of simple structural variants. 
A

Cycle of templated insertions
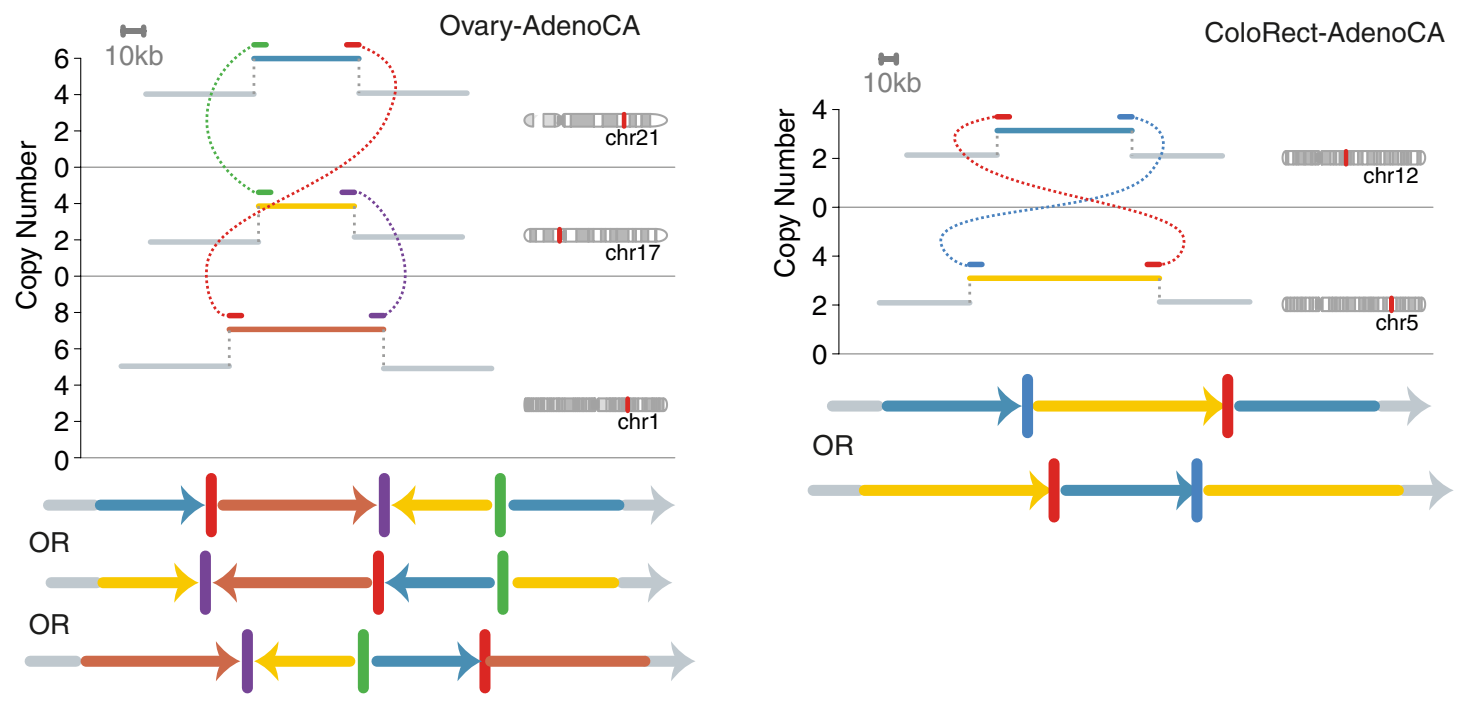

B

Bridge of templated insertions
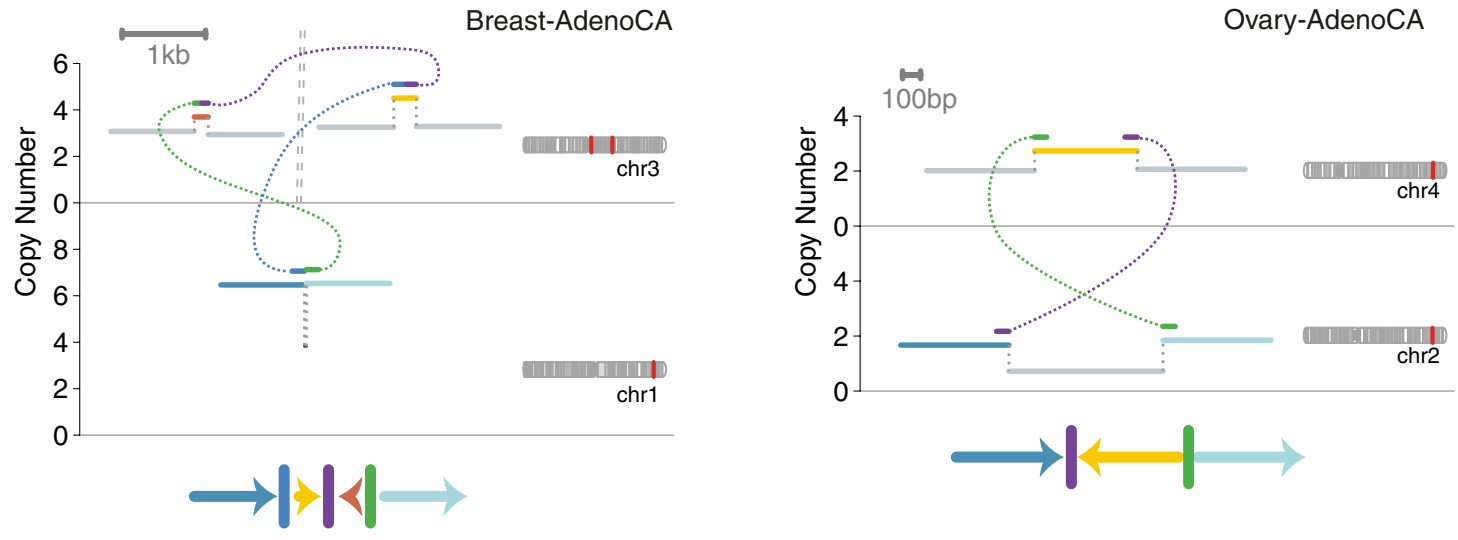

C

Chain of templated insertions
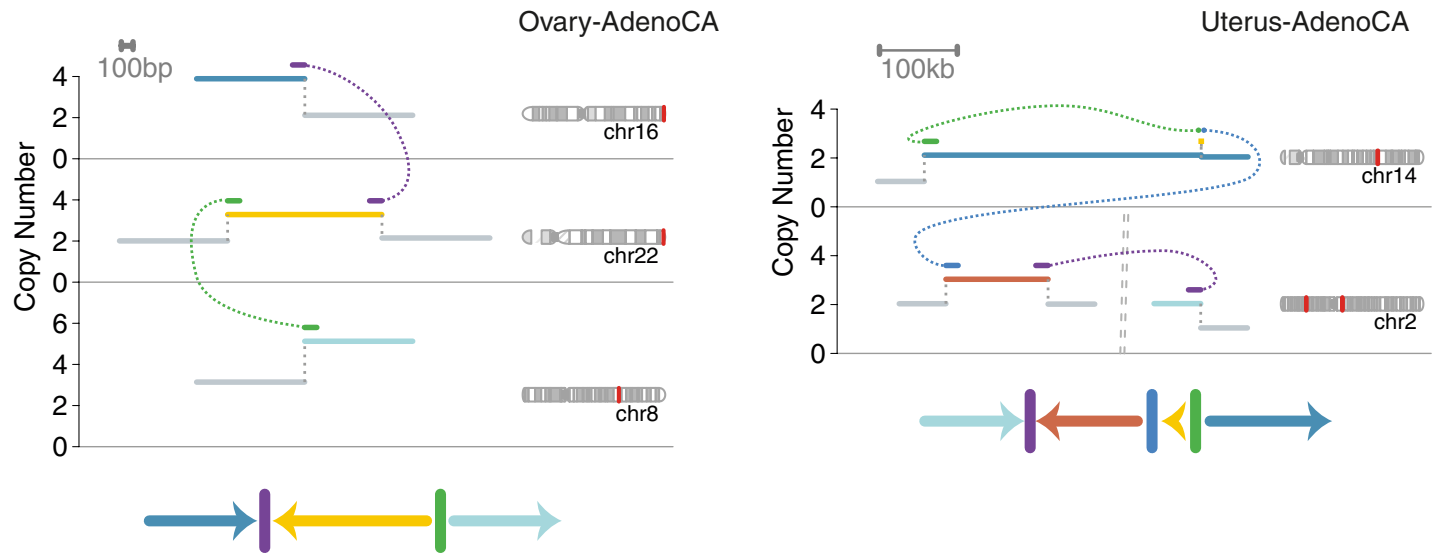

Extended Data Fig. 2 | Further examples of templated insertion chains, cycles and bridges. Schematics follow the same structure as in Fig. 3. 
A

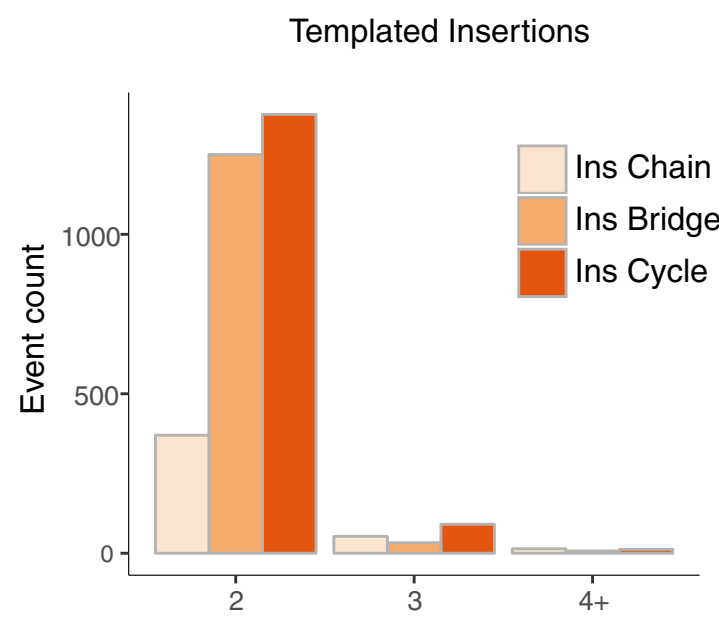

Number of breakpoint junctions

\section{Cycle of templated insertions}

One possible derivative chromosome (of 7 , depending on where cycle starts)

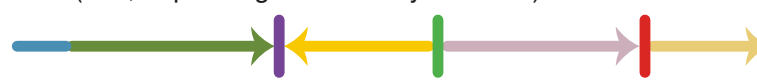

C

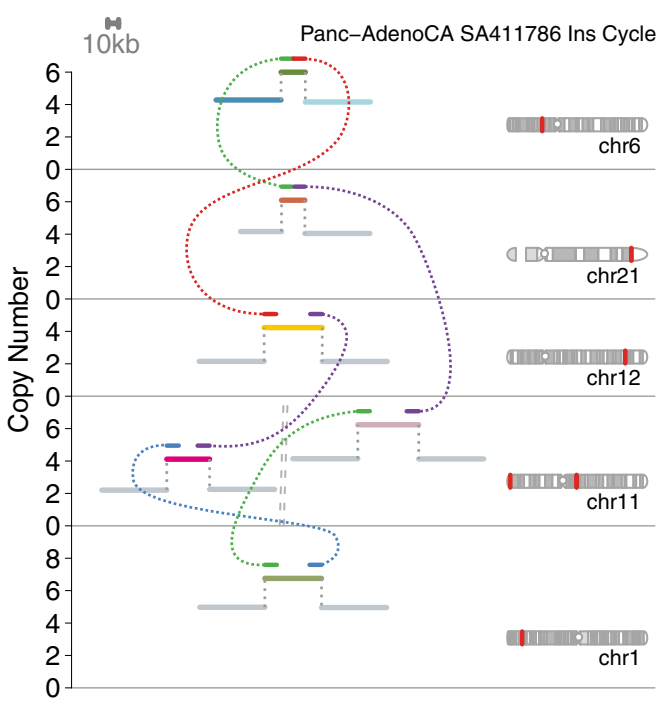

Extended Data Fig. 3 | Number of breakpoint junctions in cycles, bridges and chains of templated insertions. a, Histogram of numbers of breakpoint junctions in templated insertion cycles, chains and bridges across all samples
B $\quad \overrightarrow{100 \mathrm{~kb}} \quad$ Uterus-AdenoCA SA514439 Ins Cycle

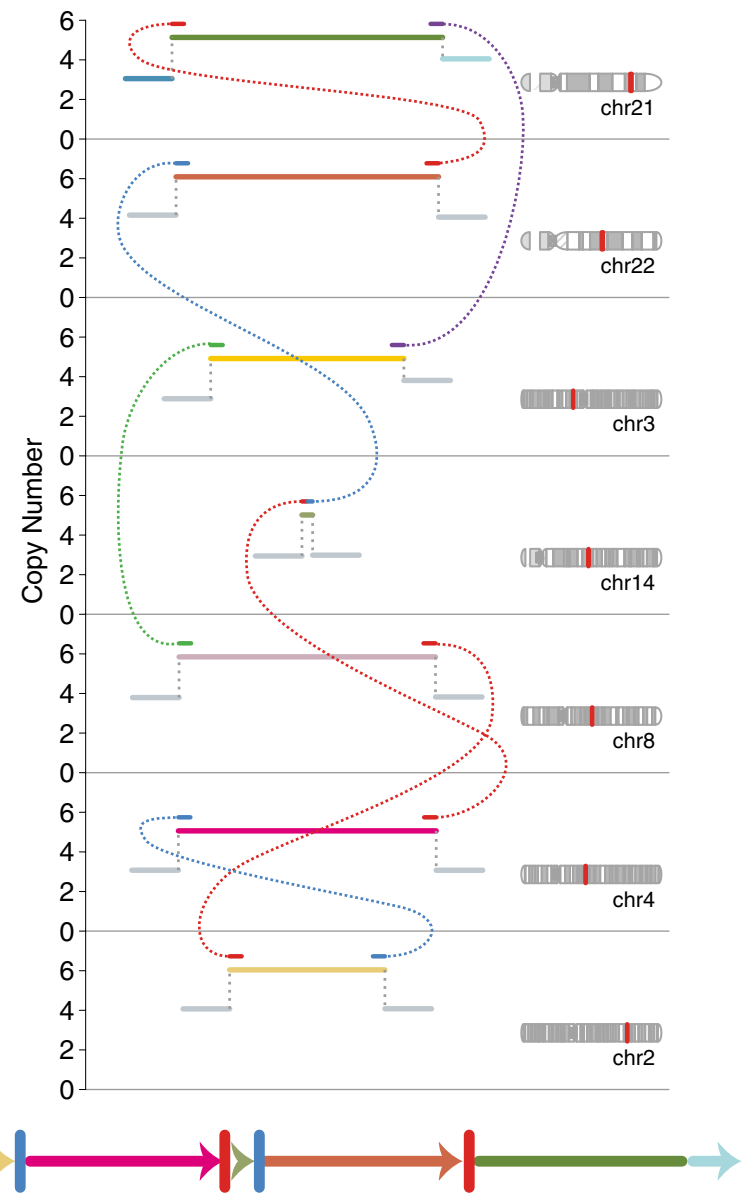

\section{Cycle of templated insertions}

One possible derivative chromosome (of 6, depending on where cycle starts)

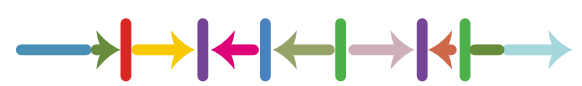

in all tumour types in the cohort.b, c, Two examples of particularly long cycles of templated insertions in the cohort. Examples are depicted in a similar manner to those in Fig. 3. 
A

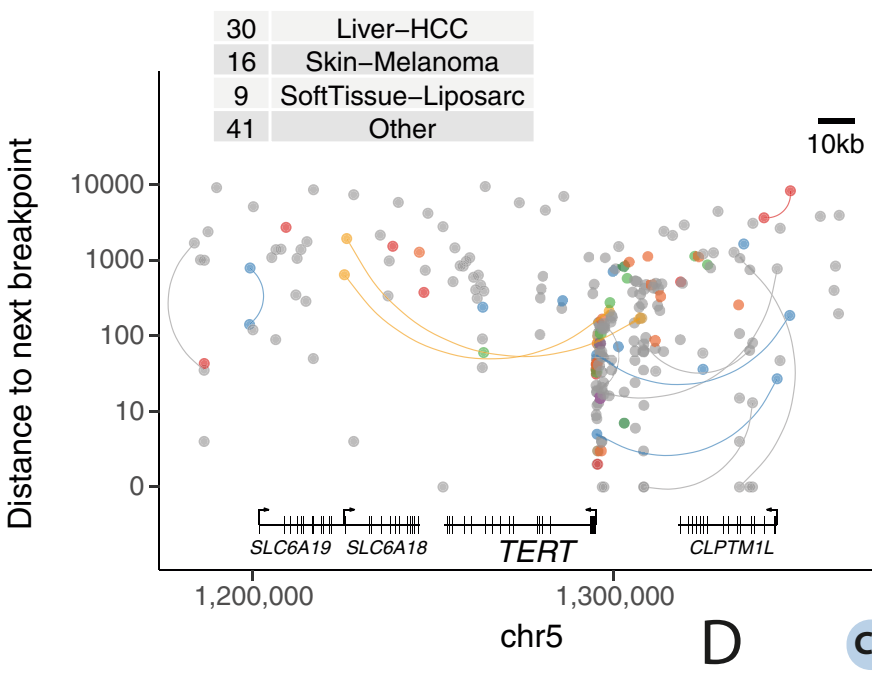

B Cycle of templated insertions

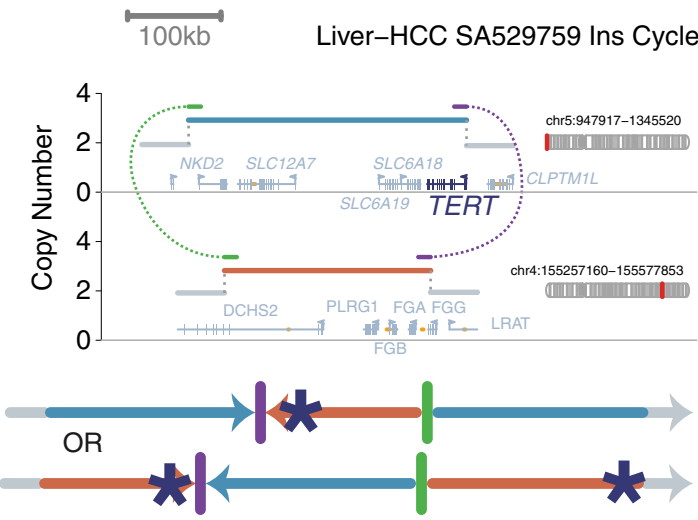

C Cycle of templated insertions
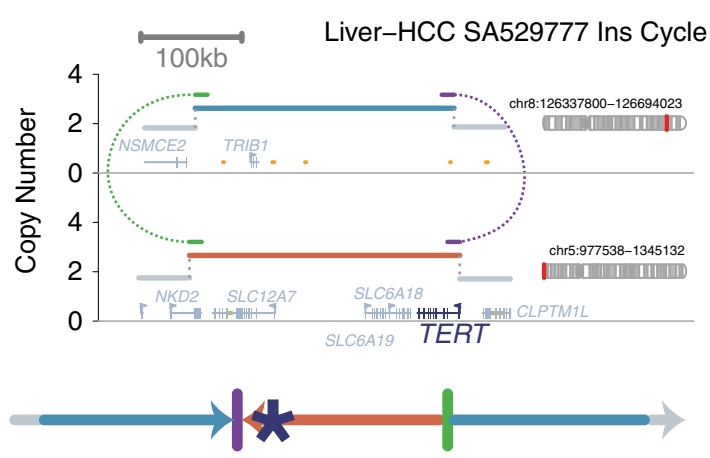

OR

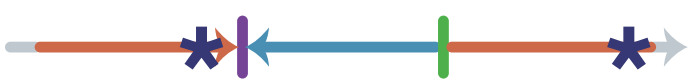

Extended Data Fig. 4 | Templated insertion events that activate TERT in hepatocellular carcinoma. a, The positions of all structural-variant breakpoints in the TERT region in the PCAWG cohort (including $50-\mathrm{kb}$ flanks either side of TERT), coloured by classification and vertically spaced by the distance to the next breakpoint in the cohort. If the two sides of a breakpoint junction are contained within the plotting window, they are joined by a curved line. The number of samples with a breakpoint in the plotting window is annotated in the table in the top left. b-d, Examples of two cycles and a chain
Complex, unclassified

- Deletion

- Tandem Duplication

Unbalanced Inversion

- Foldback inversion

- Local 2-Jump

- Reciprocal Translocation

- Unbalanced Translocation

- Reciprocal Inversion

- Templated Insertions

- Chromoplexy

- Translocation Plus (local-distant)

\section{Chain of templated insertions}
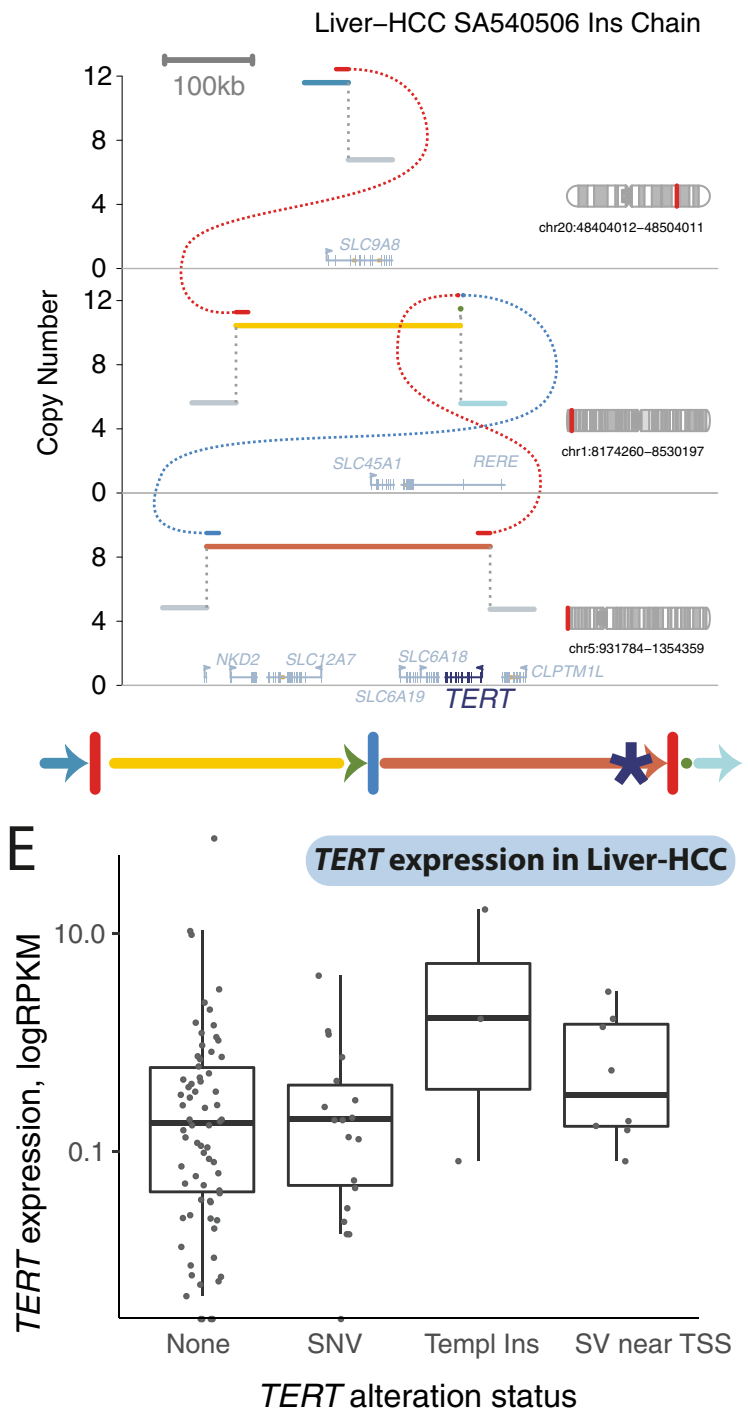

of templated insertions that affect $T E R T$ in hepatocellular carcinomas. e, Expression levels of TERT in patients with hepatocellular carcinoma ( $n=187$ patients), separated by whether TERT was wild type, had an activating promoter point mutation, structural variants in a templated insertion or other class. Individual patient data are shown as points. The box shows the median expression level as a thick black line, with the range of the box denoting the interquartile range. The whiskers show the range of data or $1.5 \times$ the interquartile range (whichever is lower). 


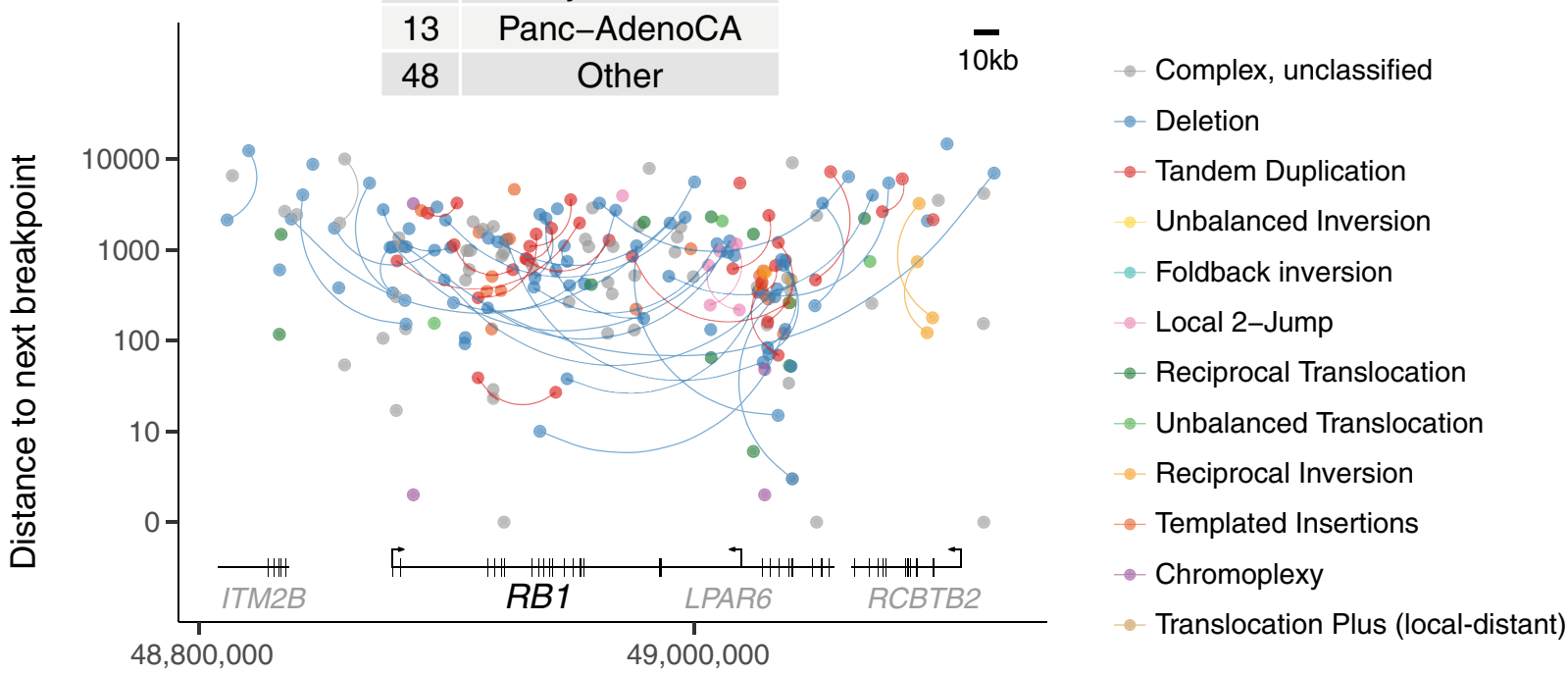

chr13

\section{B Bridge of templated insertions}

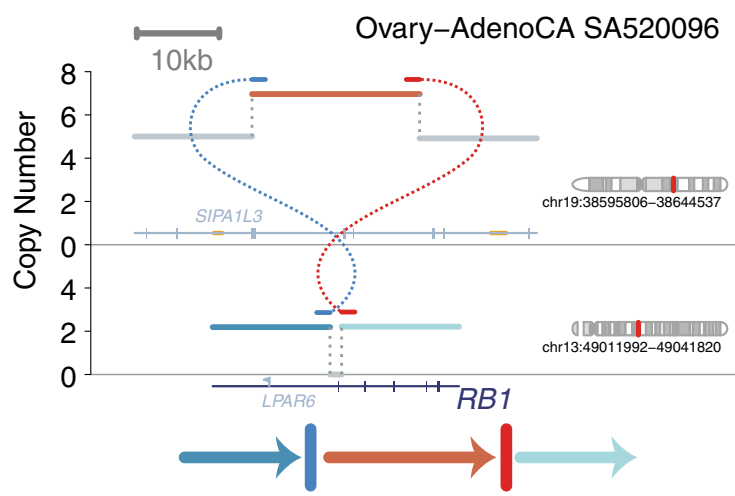

C

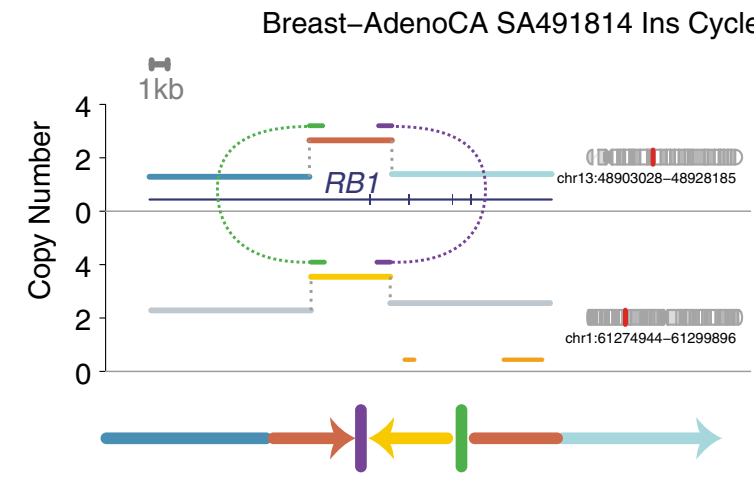

Extended Data Fig. 5 | Templated insertion events inactivating $R B 1$ in breast and ovarian carcinomas. a, The positions of all structural-variant breakpoints in the $R B 1$ region in the PCAWG cohort (including 50-kb flanks either side of $R B 1$ ), coloured by classification and vertically spaced by the distance to the next breakpoint in the cohort. If the two sides of a breakpoint

\section{Cycle of templated insertions}

Breast-AdenoCA SA18463 Ins Cycle

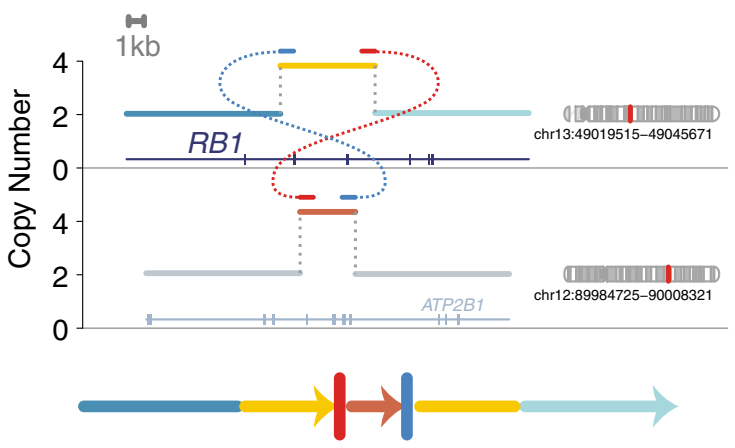

E Cycle of templated insertions

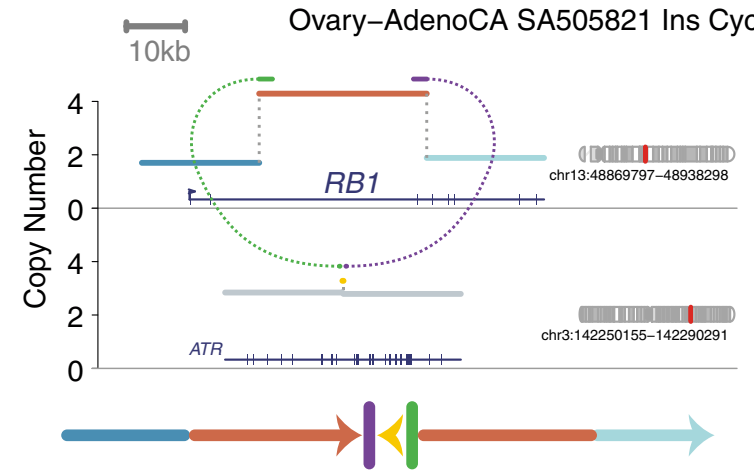

junction are contained within the plotting window, they are joined by a curved line. The number of samples with a breakpoint in the plotting window is annotated in the table in the top left. b-e, Examples of three cycles and a bridge of templated insertions that affect $R B 1$ in breast and ovarian carcinomas. 
A Tandem Duplication Size by tumour type
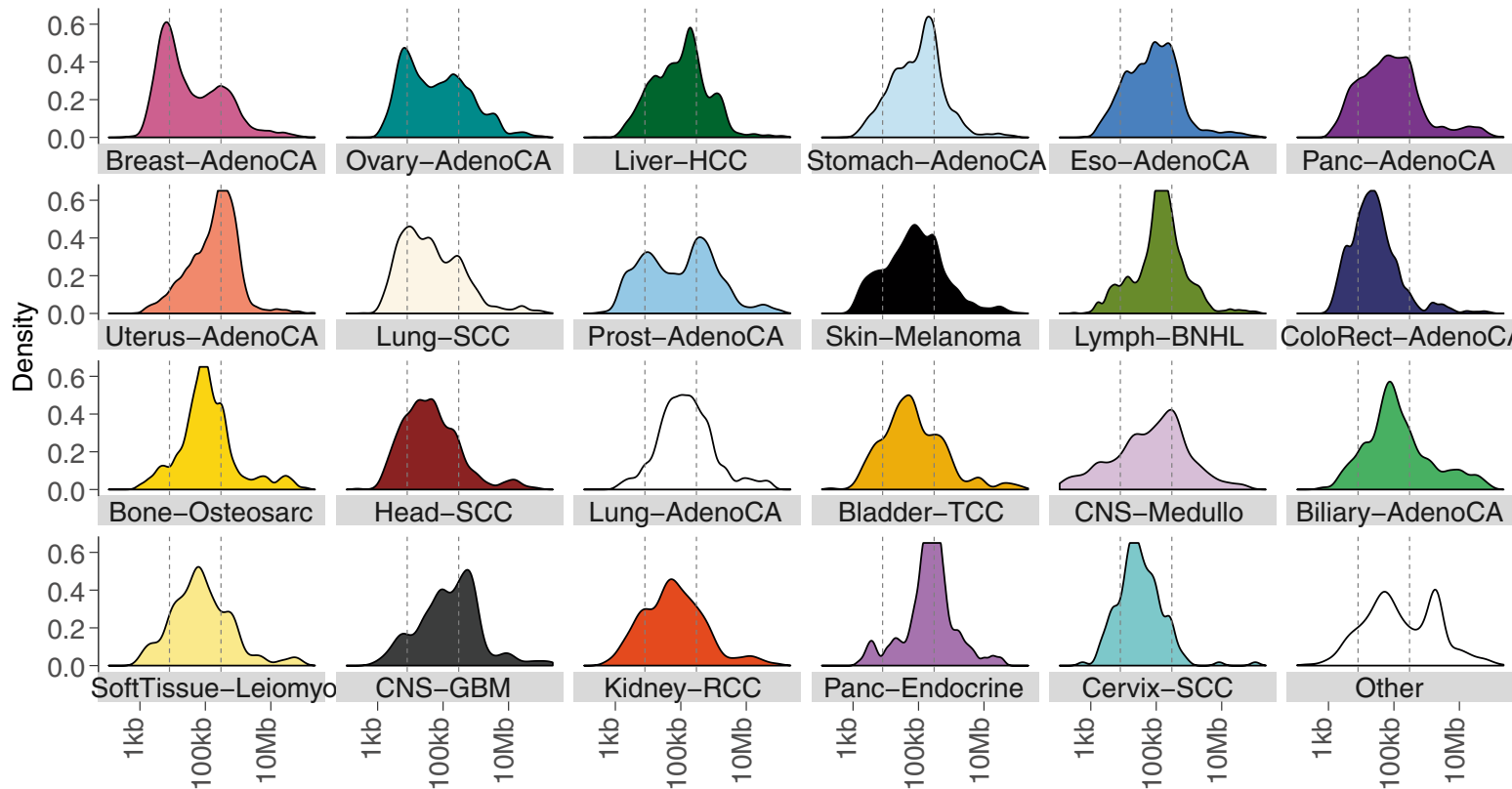

B Tandem duplication size in individual samples

\section{Sample median}

$1 \mathrm{~kb} \quad 10 \mathrm{~kb} \quad 100 \mathrm{~kb} \quad 1 \mathrm{Mb}$

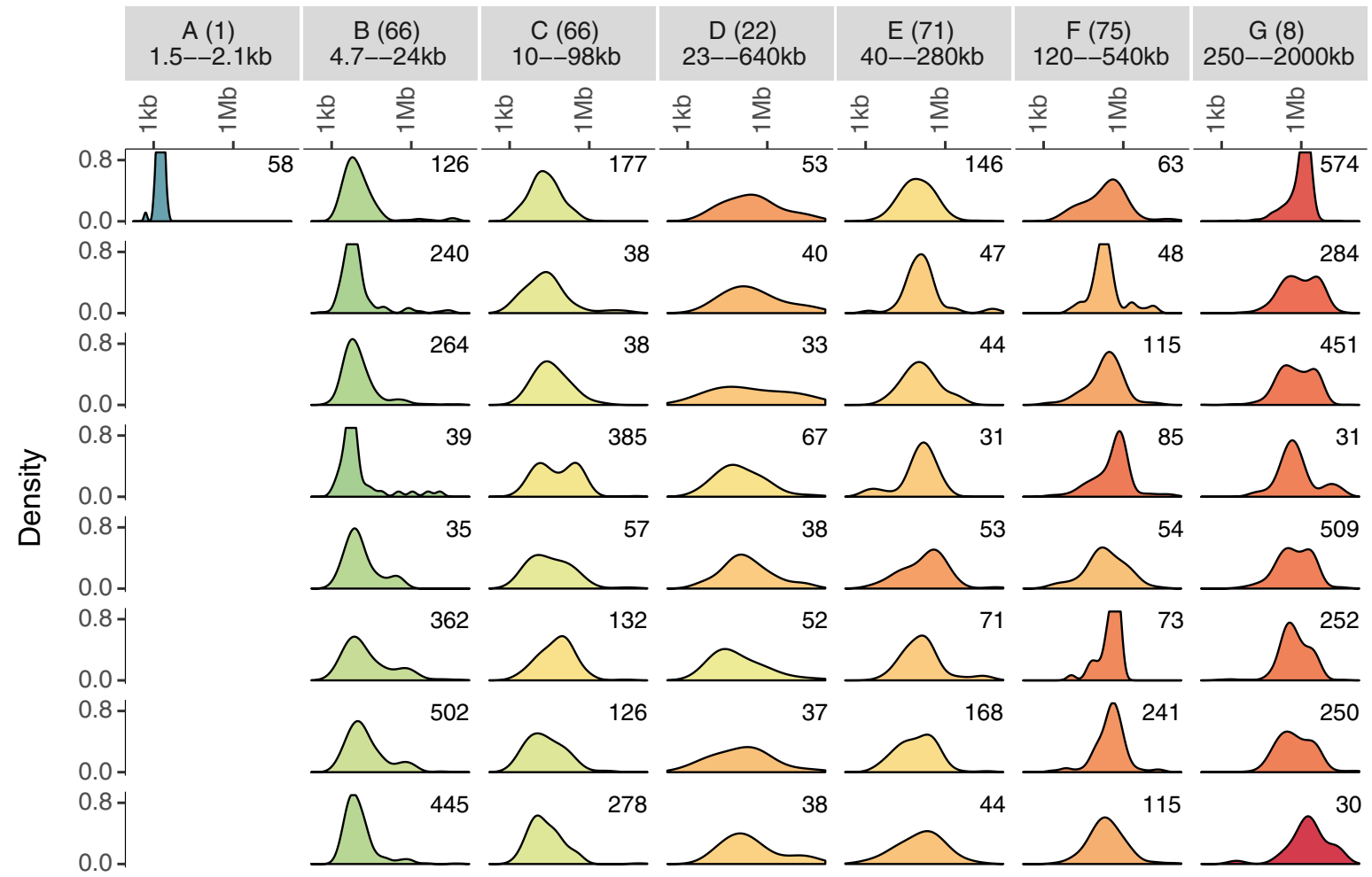

Extended Data Fig. 6 | Size distribution of tandem duplications. a, Size distribution of tandem duplications per histology group. b, Samples with more than 20 tandem duplications were grouped using hierarchical clustering according to the within-patient distribution of tandem-duplication size. Seven clusters emerged, with the size distribution of up to eight randomly chosen samples per cluster illustrated. The numbers in the top right of each panel denote the number of tandem duplications in that sample. 

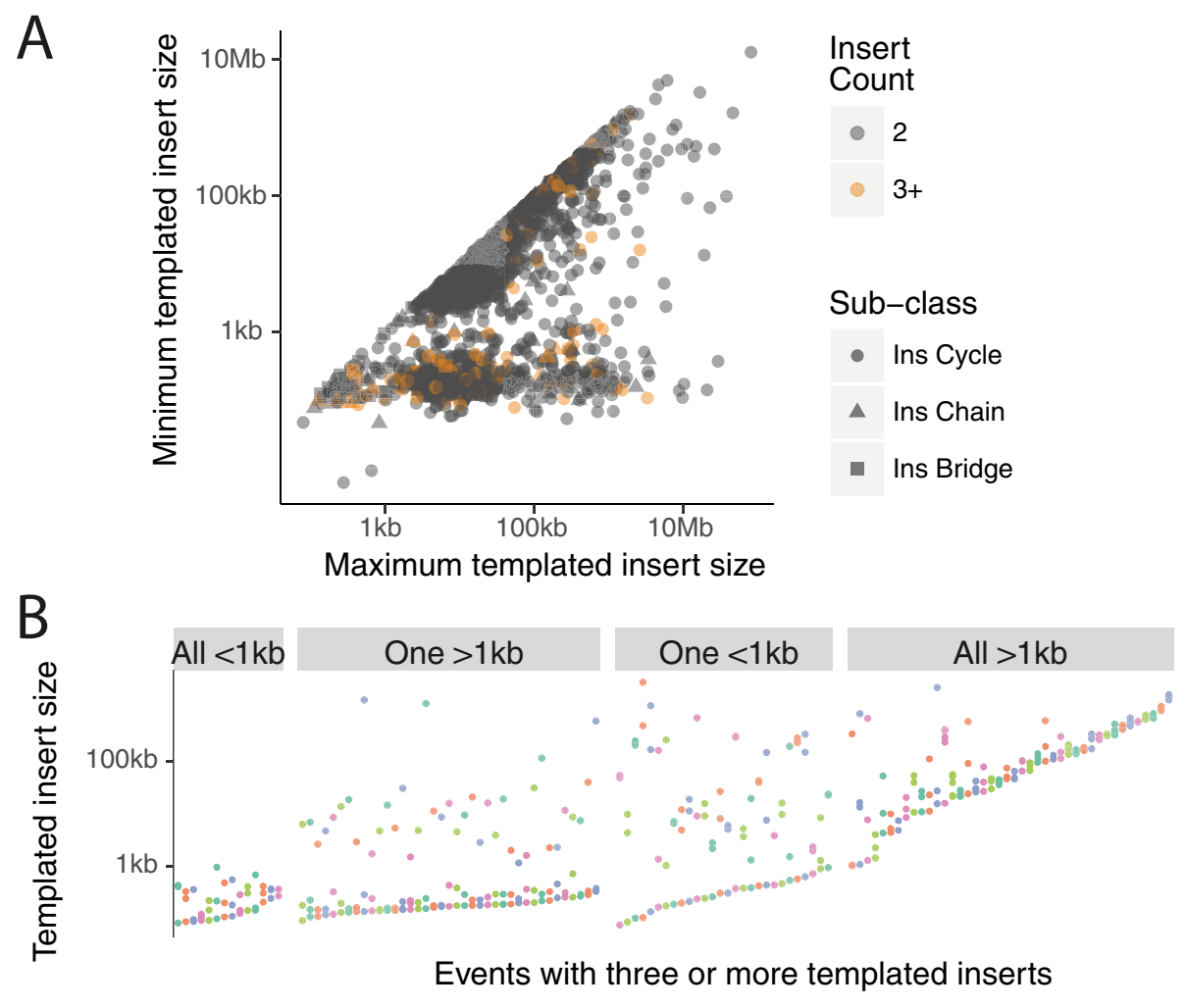
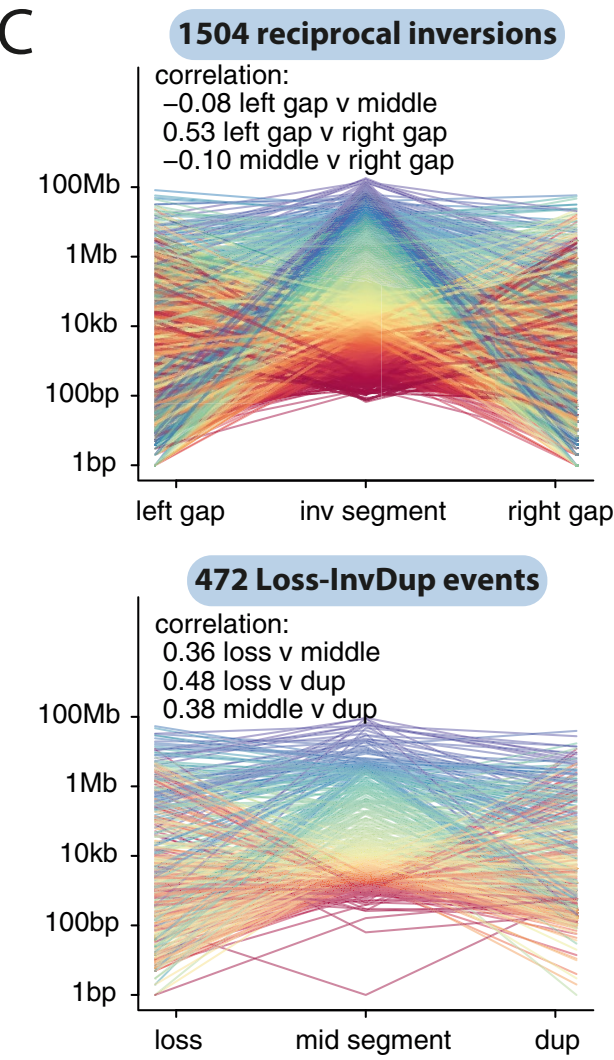

Extended Data Fig. $7 \mid$ Size properties of clustered structural-variant classes. a, Comparison of the minimum and maximum templated-insert size for multi-insert cycles, chains and bridges of templated insertions. $\mathbf{b}$, All events with three or more templated inserts, grouped by combination of insert sizes. c, Correlations (Pearson's correlation coefficient) and raw sizes of individual

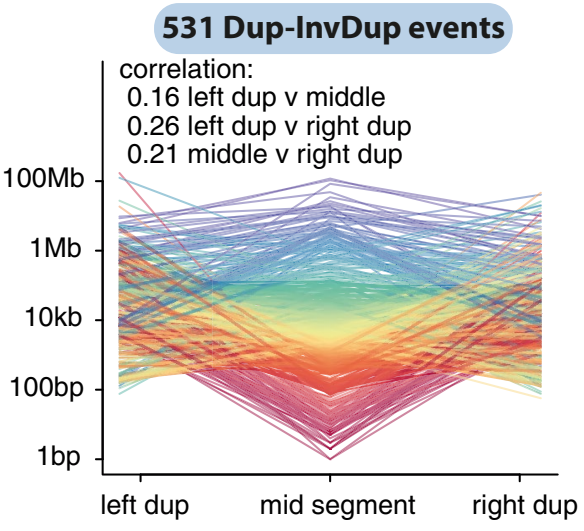

\section{Dup-Trp-Dup events}

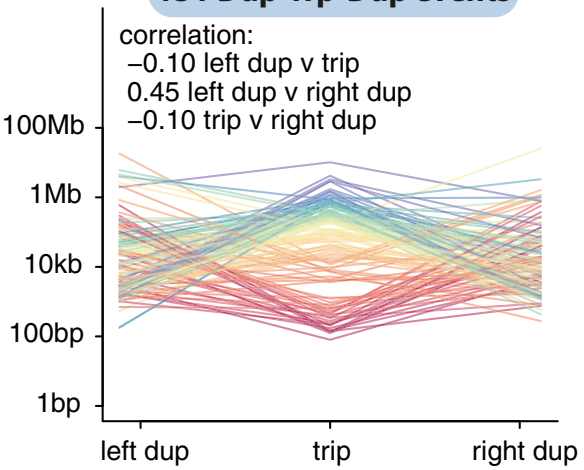

genomic segments for reciprocal inversions and local two-jumps. Each individual event is shown as a line that links the size of the individual segments in that event. The sample sizes for each event class are shown in the labels for each panel. 


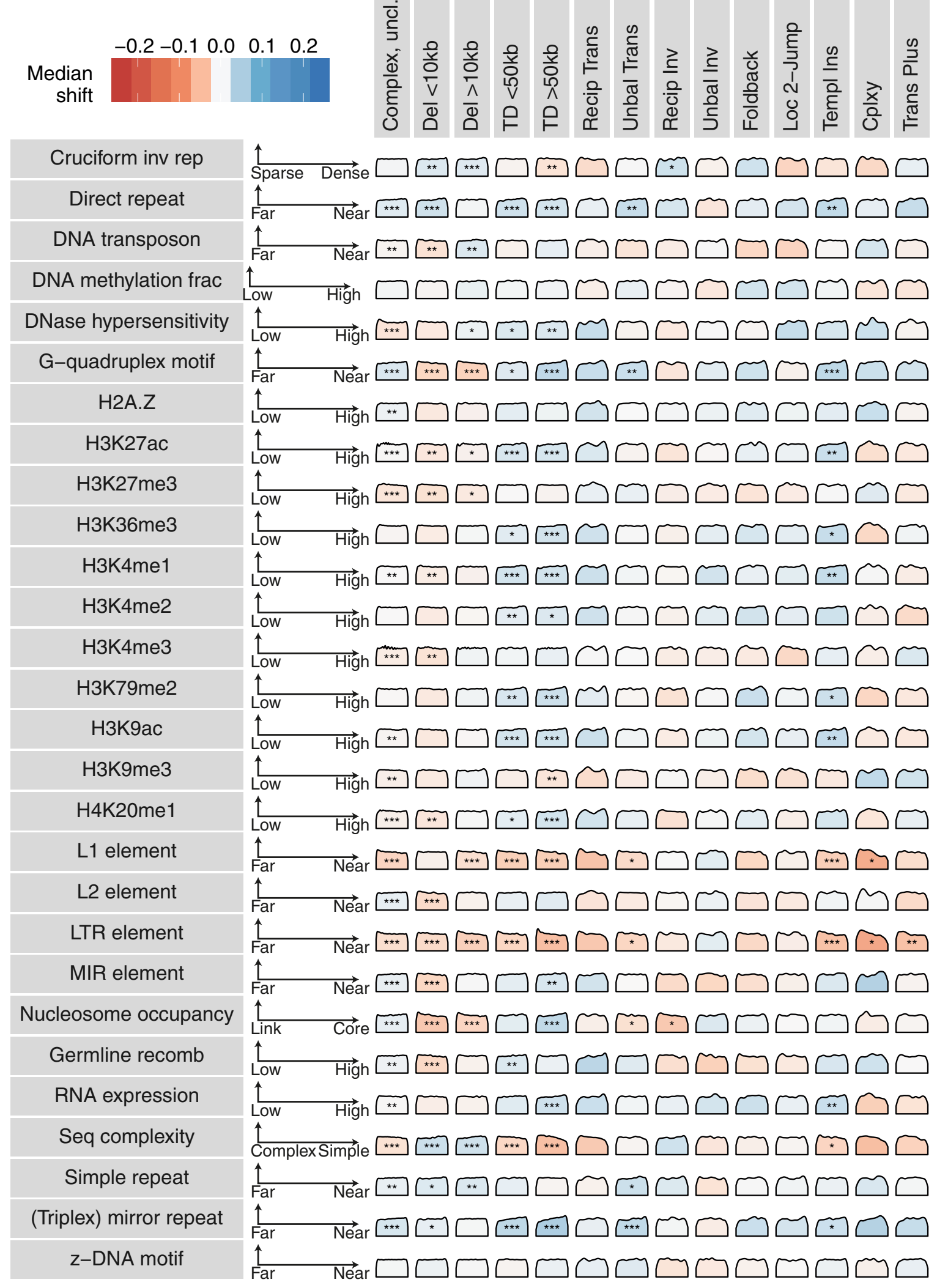

Extended Data Fig. 8 | Relationship of an extended panel of genomic properties with structural-variant categories. Associations between a subset of the genomic properties (rows) and classes of structural variant (columns). Each density curve represents the quantile distribution of the genomic property values at observed breakpoints, compared to random genome positions. Asterisks indicate significant departures from uniform quantiles after multiple hypothesis correction by the Benjamini-Yekutieli method on a one-sided Kolmogorov-Smirnov test, based on a sample size of 2,559 genomes containing structural variants: ${ }^{*}$ false-discovery rate $<0.01,{ }^{* *}$ false-discovery rate $<0.001,{ }^{* * *}$ false-discovery rate $<10^{-6}$. Cells with significant property associations are shaded by the magnitude of the shift of the median observed quantile above (blue) or below (red) 0.5 . The interpretation of each property from left to right is indicated by the axes to the right of the property label. 
A FRA3B FHIT

\section{FRA20B MACROD2}

FRA16D WWOX
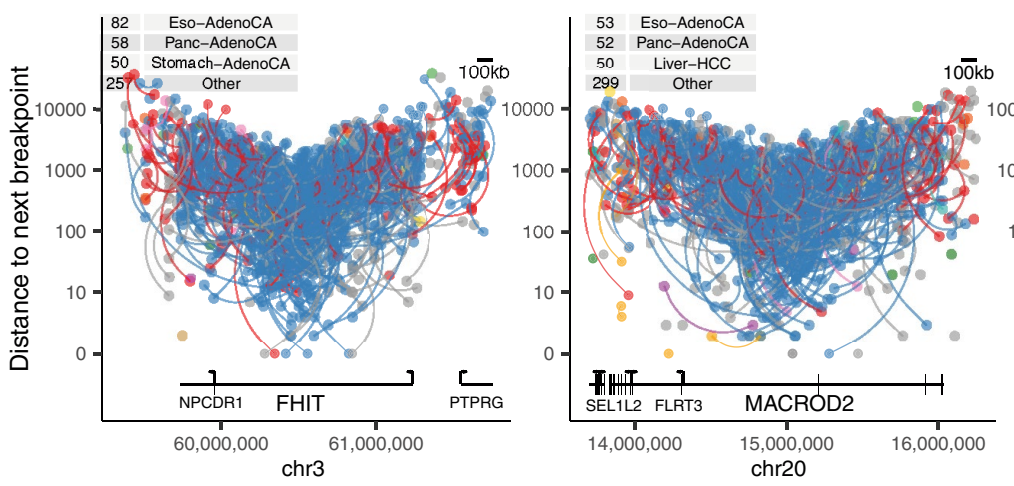

$\rightarrow$ Complex, unclassified $\rightarrow$ Unbalanced Inversion
$\rightarrow$ Deletion
$\rightarrow$ Tandem Duplication $\rightarrow$ Foldback inversion

- Reciprocal Translocation

- Unbalanced Translocation

$\rightarrow$ Reciprocal Inversion

B

B

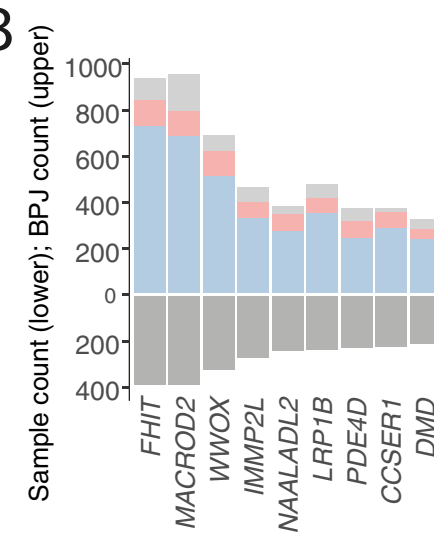

C

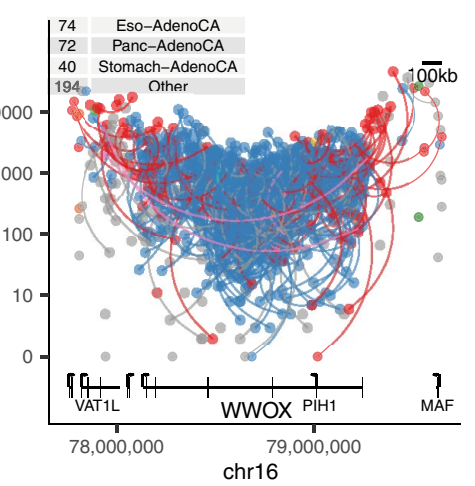

- Templated Insertions

- Chromoplexy

- Translocation Plus (local-distant)

Other

Not in FS $\square$ In FS

Tandem Dup

Deletion

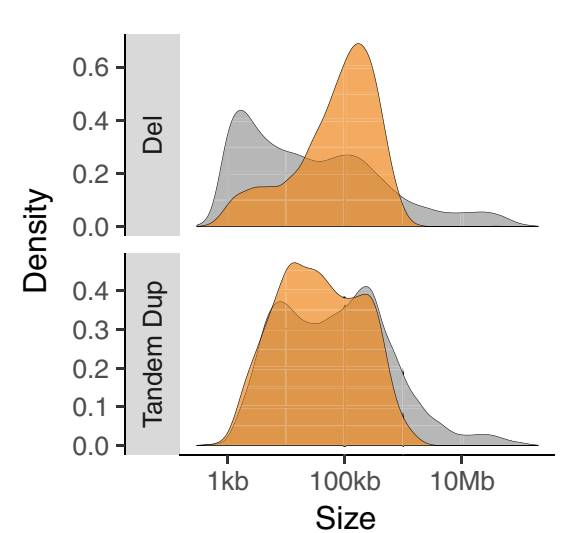

D

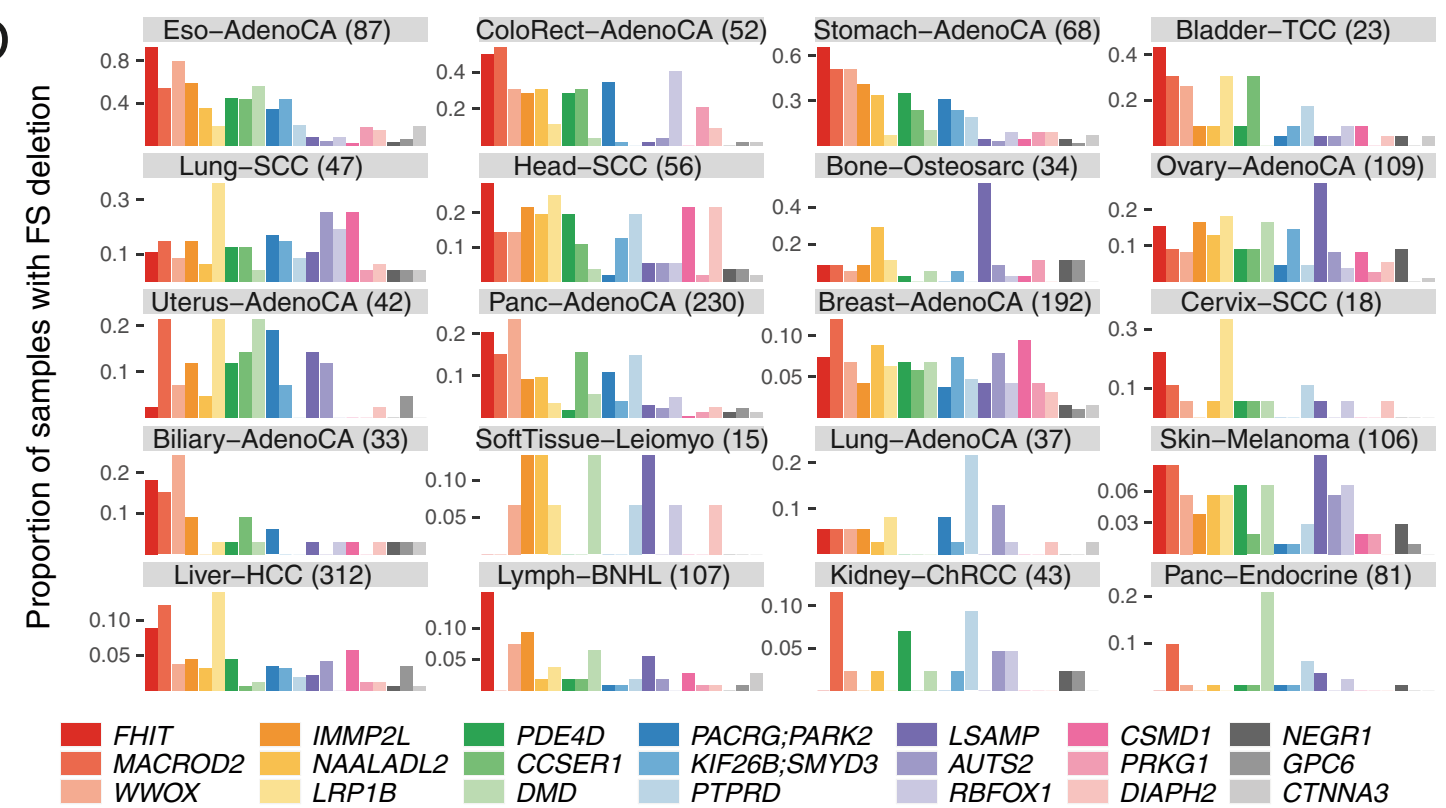

Extended Data Fig. 9 | Properties of structural variants at chromosomal fragile sites. a, Structural-variant breakpoints in the most affected fragile sites: FHIT, MACROD2 and WWOX. These are coloured by classification and vertically spaced by the distance to the next breakpoint in the cohort. If the two sides of a breakpoint junction are contained within the plotting window, they are joined by a curved line. The number of samples with a breakpoint in the plotting window is annotated in the tables at the top left. b. Number of deletions and tandem duplications (top) and number of affected samples (bottom) for the 18 fragile sites considered in this analysis.c, Size distribution of deletions and tandem duplications in fragile sites (FS) compared to the rest of the genome. d, Fragile-site preference for 20 cancer histology groups as indicated by the proportion of samples that contains a deletion in each of the 18 fragile sites considered here. The number of samples is indicated in parentheses. 


\section{A}

Small del SV signature vs BRCA2 mutation

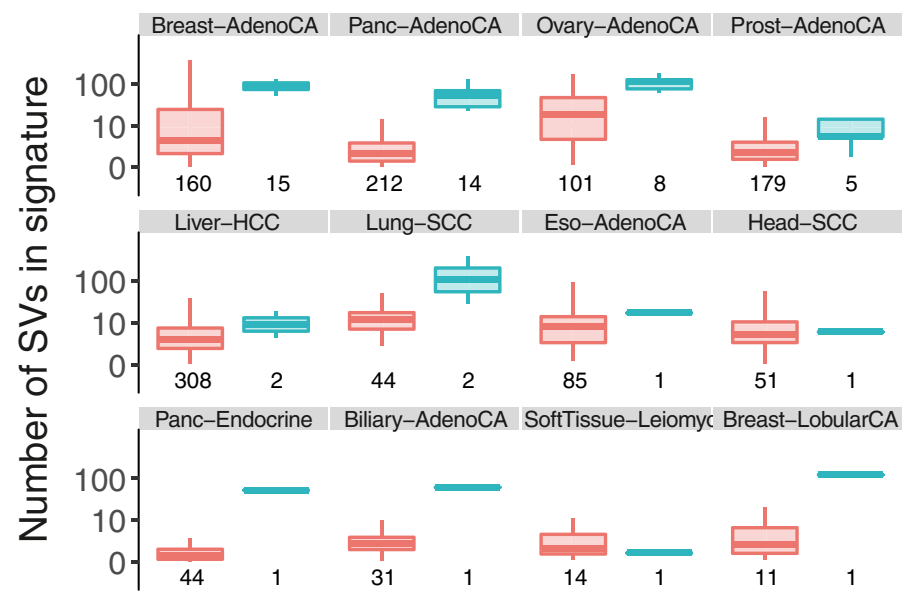

BRCA2

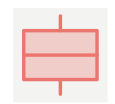

FALSE

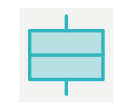

TRUE

\section{B}

\section{Small del SV signature vs PALB2 mutation}

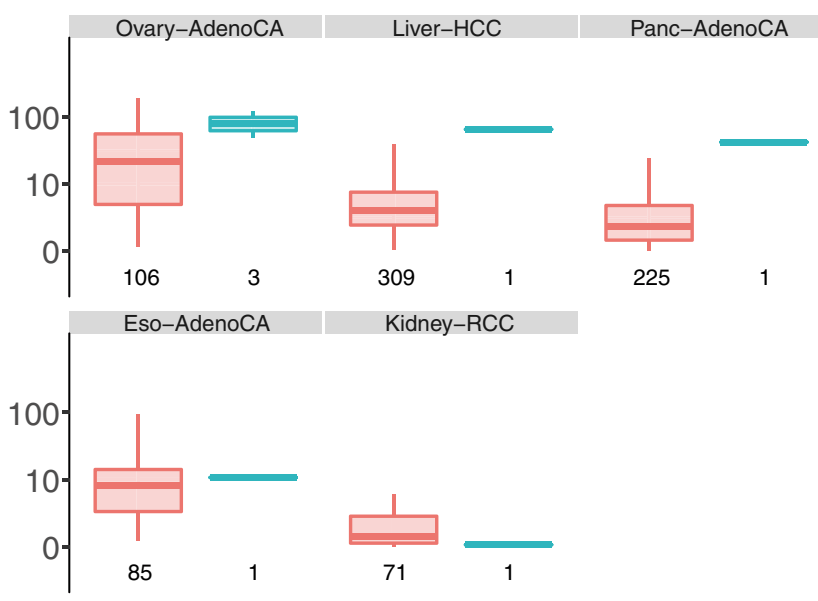

PALB2

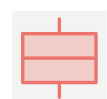

FALSE

TRUE
C

Early, small TD SV signature vs BRCA1 mutation

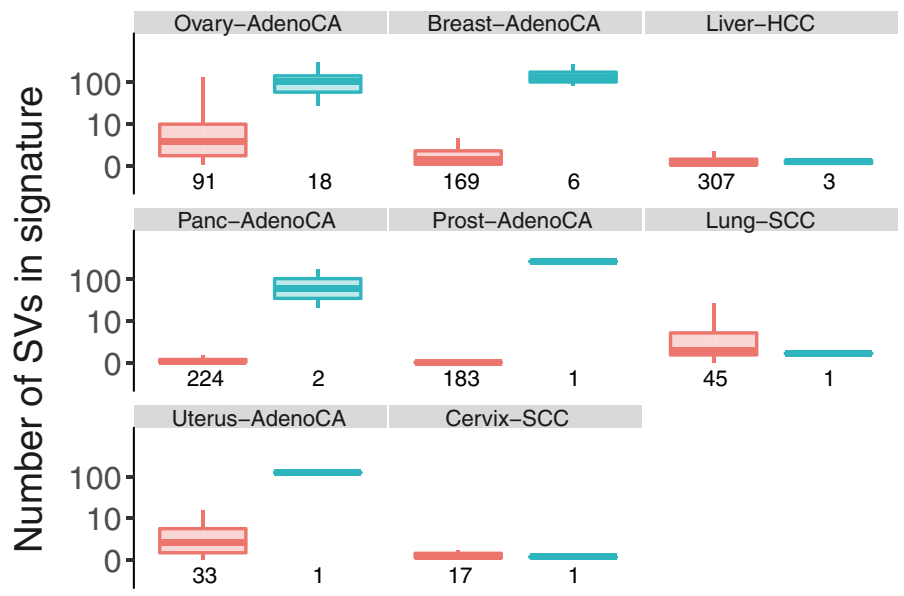

BRCA1

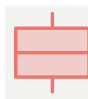

FALSE

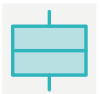

TRUE

Extended Data Fig. 10 | Consistency of associations between signatures and mutations in DNA-repair genes. a, Box-and-whisker plots showing the number of structural variants attributed to the small-deletion signature in different types of tumour, split by $B R C A 2$ status (BRCA2 wild type in orange; $B R C A 2$ mutant in cyan). The box denotes the interquartile range, with the median marked as a horizontal line. The whiskers extend as far as the range or $1.5 \times$ the interquartile range, whichever is lower. Outlier patients are shown as points. There is an increase in events attributed to the small-deletion signature when $B R C A 2$ is mutated, across multiple types of tumour (breast, pancreatic,
D

\section{Large TD SV signature vs CDK12 mutation}
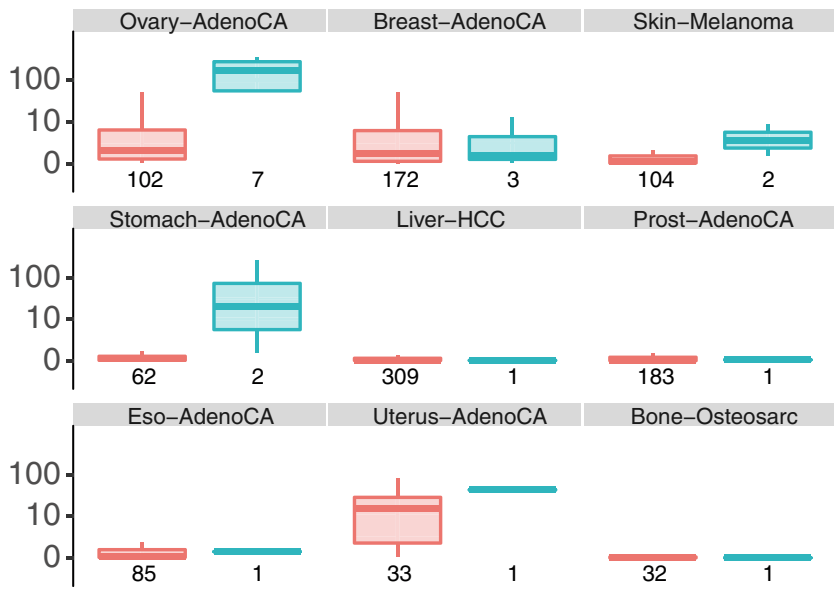

CDK12

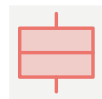

FALSE

TRUE

ovarian, prostate, lung squamous and so on). b, Box-and-whisker plots as for a, showing the number of structural variants at tributed to the small-deletion signature in different types of tumour, split by PALB2 status.c, Box-and-whisker plots as for a, showing the number of structural variants attributed to the earlyreplicating, small-tandem-duplication signature in different types of tumour, split by BRCA1 status. d, Box-and-whisker plots as for a, showing the number of structural variants attributed to the large-tandem-duplication signature in different types of tumour, split by $C D K 12$ status. 

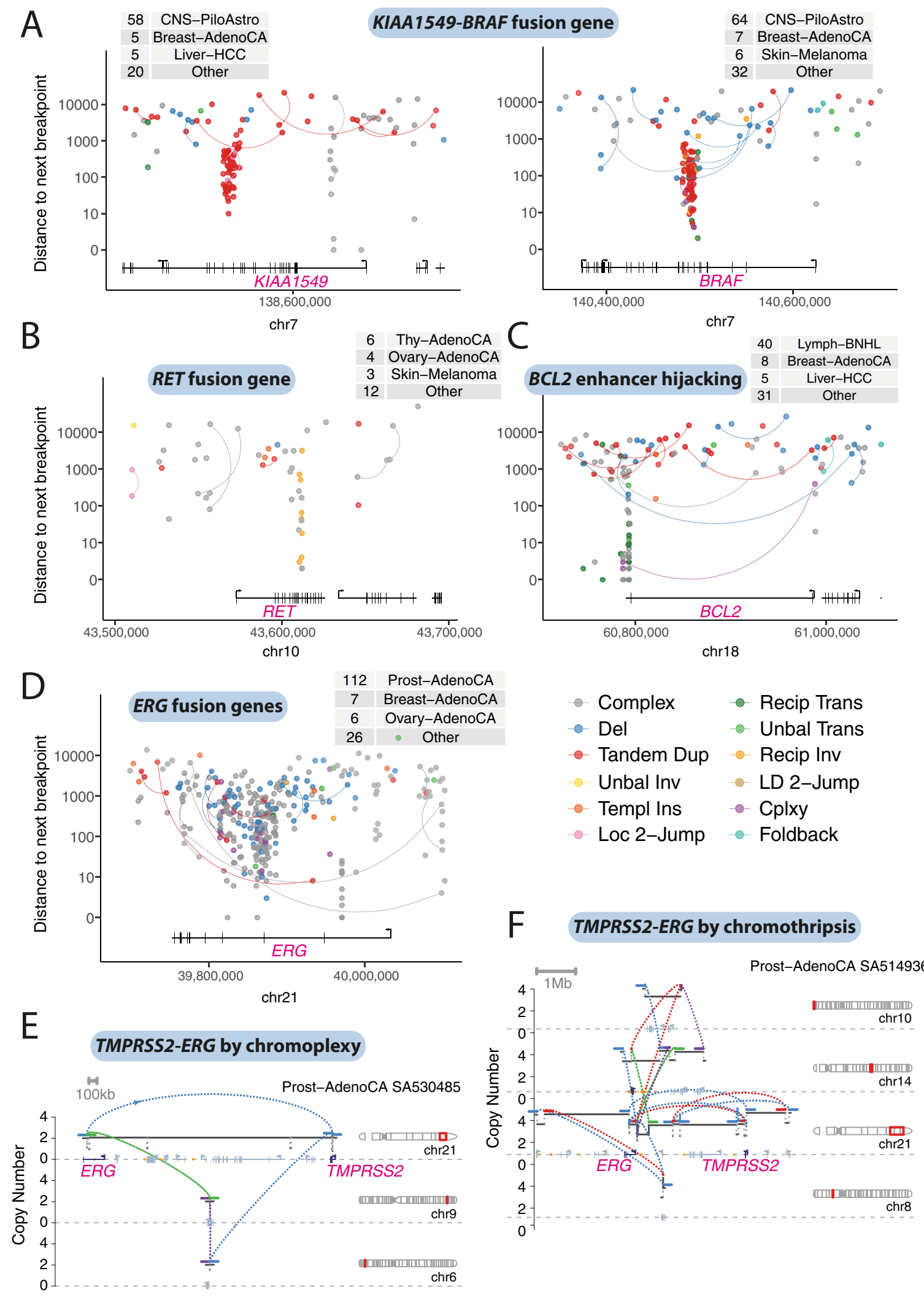

Extended Data Fig. 11 |See next page for caption. 
Extended Data Fig. 11 | Patterns of structural variants causing fusion genes and enhancer hijacking. a, Rainfall plot of structural-variant breakpoints in the genes KIAA1549 and $B R A F$, commonly fused together through a tandem duplication in pilocytic astrocytomas. Structural variants are coloured by classification and arranged vertically by the distance to the next breakpoint in the cohort. If the two sides of a breakpoint junction are contained within the plotting window, they are joined by a curved line. The number of samples with a breakpoint in the plotting window is annotated in the table at the top of each panel.b, Rainfall plot of structural-variant breakpoints that affect $R E T$, commonly fused to $C C D C 6$ by inversion in papillary thyroid cancer. $\mathbf{c}$, Rainfall plot of structural-variant breakpoints that affect $B C L 2$, commonly hijacked to the $I G H$ immunoglobulin locus by translocations in B cell lymphomas. d, Rainfall plot of structural-variant breakpoints that affect $E R G$, commonly fused with TMPRSS2 by deletion or more-complex events in prostate adenocarcinoma.e, Example of a TMPRSS2-ERG fusion gene in a prostate adenocarcinoma created by a chromoplexy cycle. The estimated copy-number profile is shown as black horizontal segments, with structural variants shown as dotted arcs linking the edges of two copy-number segments. f, Example of a TMPRSS2-ERG fusion gene in a prostate adenocarcinoma created by chromothripsis. 

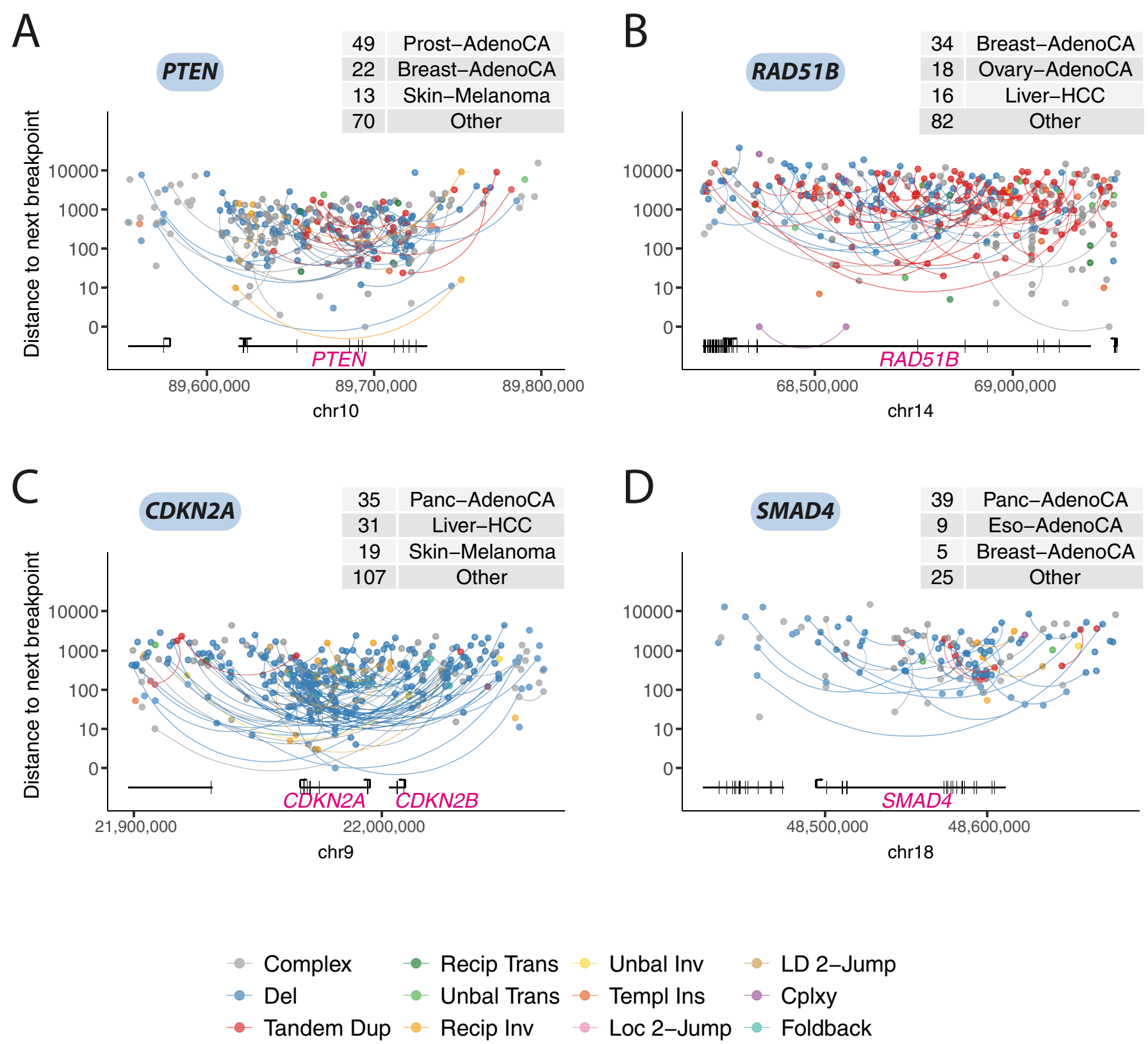

Extended Data Fig. 12 Patterns of structural variants that affect selected tumour-suppressor genes. a, Rainfall plot of structural-variant breakpoints in the gene PTEN, commonly inactivated in breast and ovarian adenocarcinomas, in which tandem-duplication signatures are frequent. Structural variants are coloured by classification and arranged vertically by the distance to the next breakpoint in the cohort. If the two sides of a breakpoint junction are contained within the plotting window, they are joined by a curved line. The number of samples with a breakpoint in the plotting window is annotated in the table at the top of each panel. b, Rainfall plot of structural-variant breakpoints that affect $R A D 51 B$, commonly inactivated in breast and ovarian adenocarcinomas. c, Rainfall plot of structural-variant breakpoints that affect $C D K N 2 A$, commonly inactivated in tumours of the gastrointestinal tract, in which deletion signatures are common. d, Rainfall plot of structural-variant breakpoints that affect SMAD4, commonly inactivated in tumours of the gastrointestinal tract. 


\section{Reciprocal translocation}

$1 \mathrm{~kb}$

Lymph-BNHL SA320992

\section{Local 3-jump}

100kb Breast-AdenoCA SA6128

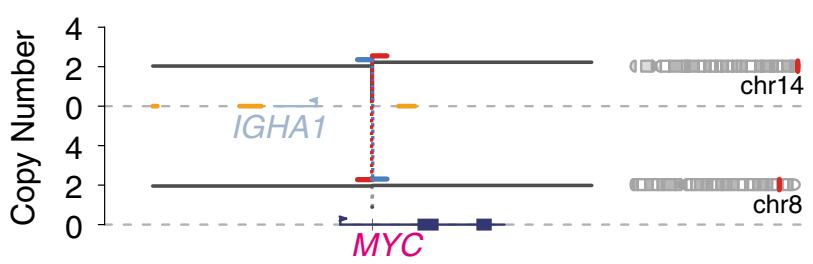

Cycle of templated insertions

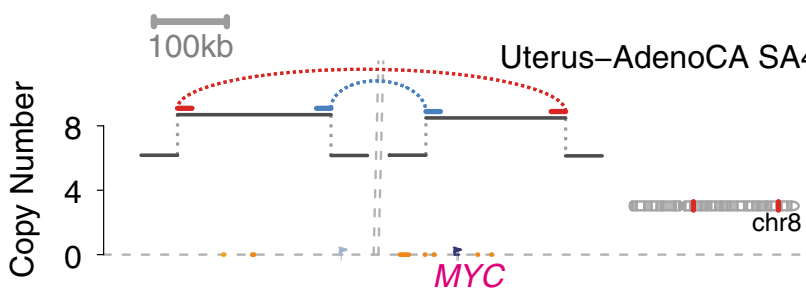

Local-distant cluster
$10 \mathrm{~kb}$
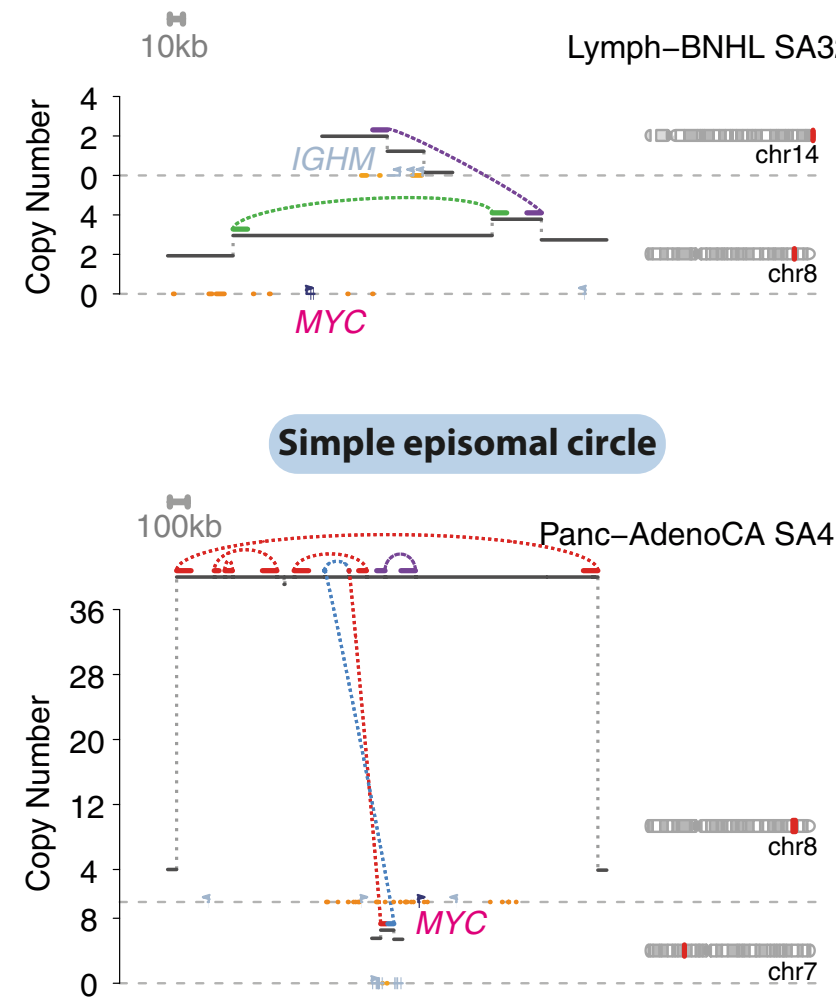

\section{Simple episomal circle}

$00 \mathrm{~kb}$
Complex intrachromosomal amplification

$\stackrel{\mathrm{Mb}}{\mathrm{Mb}}$

\section{Nested tandem duplications}

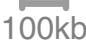

Stomach-AdenoCA SA444038

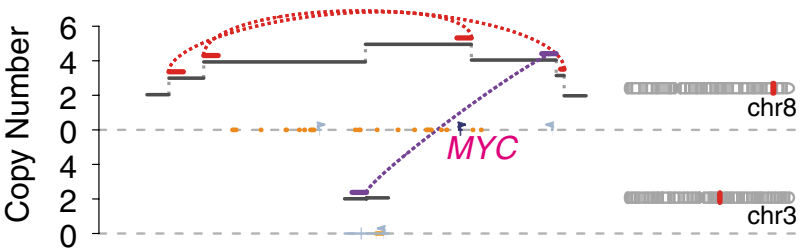

c-AdenoCA SA411002
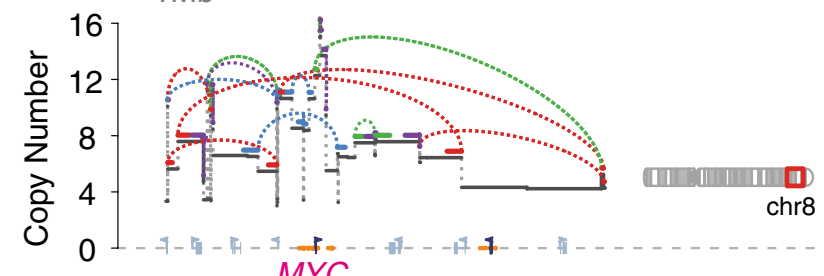

\section{Chromothripsis creating episomal circle}

$\stackrel{\longmapsto}{100 \mathrm{~kb}}$

CNS-Medullo SA517281

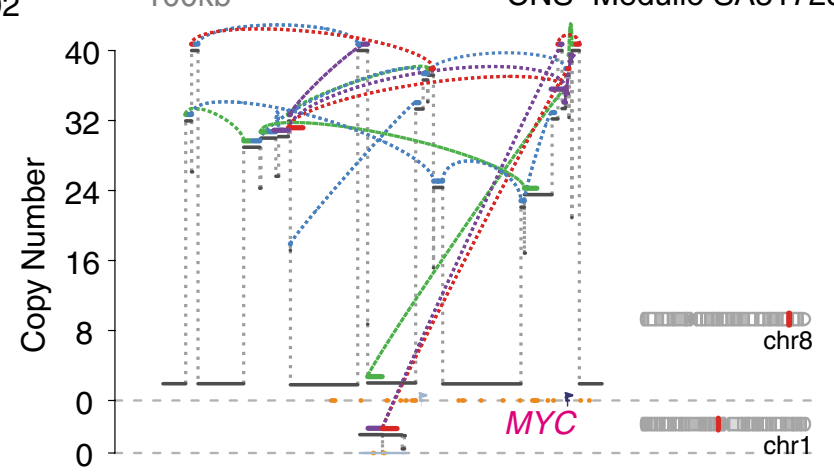

Extended Data Fig. 13 | Examples of structural variants increasing the copy number of $M Y C$. The estimated copy-number profile is shown as black horizontal segments, with structural variants shown as dotted arcs linking the edges of two copy-number segments. 


\section{Extended Data Table 1 | Glossary of key terms}

\begin{tabular}{ll}
\hline Term & Description \\
\hline Structural variant (SV) & $\begin{array}{l}\text { Juxtaposition of non-contiguous chromosomal segments through a process of genomic } \\
\text { rearrangement. } \\
\text { Breakpoint }\end{array}$ \\
$\begin{array}{l}\text { The chromosomal position at which a DNA break is made. Each SV consists of a junction } \\
\text { between two breakpoints in different regions of the genome. }\end{array}$ \\
Copy number & Change in the number of copies of a given chromosomal segment from that expected.
\end{tabular}
alteration (CNA)

Reciprocal or balanced A pair of SVs in which both sides of a single dsDNA break are rescued in the SV rearrangement. Typically used to describe some inversions and some translocations.

Unbalanced SV An SV (usually inversion or translocation) in which only one side of the dsDNA break is rescued, thereby generating a copy number alteration across the breakpoint.

Cluster of SVs A set of SVs that are closer together in genomic space than expected by chance. Typically, such clustering implies a shared mechanistic basis for the SV generation.

Derivative

chromosome

A chromosome that carries one or more SVs.

Phased SVs

Set of SVs and copy number alterations in a cluster carried on a single derivative chromosome.

Chromosomal A contiguous stretch of DNA that is of constant copy number, used to denote the regions segment of chromosome between SVs.

Template A region of chromosomal DNA that is copied and inserted elsewhere in the genome.

\section{SV class} A type of structural variant, such as deletion, tandem duplication or translocation.

Deletion Loss of a segment of chromosome from the genome spanned by a junction between the two breakpoints either side.

Tandem duplication

Extra copy of a segment of chromosome in which the duplicated region is inserted immediately adjacent to the original template in the same orientation.

Reciprocal inversion

A segment of chromosomal DNA inserted into its original position, but in the opposite orientation.

Fold-back inversion

An inverted rearrangement between two breakpoints typically $<20 \mathrm{~kb}$ apart on the chromosome, with associated copy number change. Often a sign of breakage-fusionbridge cycles.

\section{Translocation}

Breakpoint junction between two different chromosomes, either reciprocal or unbalanced.

Breakage-fusionbridge cycle

SV mechanism in which a naked DNA end (breakage) is copied to its sister chromatid during $S$ phase, with the two ends undergoing fusion (by fold-back inversion). At anaphase, the resulting dicentric chromosome is stretched between the two daughter cells (bridge), leading to further DNA breakage and potentially further cycles.

Chromoplexy

A set of $>2$ reciprocal SVs in which the chromosomal ends either side of each breakpoint are shuffled such that every end is rescued in a rearrangement junction.

Chromothripsis

A cluster of many SVs (10s to 100s) in one or a few chromosomes, occurring in a single catastrophic event, with oscillating copy number profile and rearrangement junctions of all four possible orientations.

Local $n$-jump

A cluster of $n$ SVs in a single genomic region, typically phased to a single derivative chromosome, exhibiting some copy number gains and junctions with inverted and noninverted orientation.

Cycle, chain or bridge Copies of one or more genomic templates drawn from across the genome, strung together of templated insertions in a contiguous string and inserted into a single derivative chromosome. A chain of templated insertions does not return to the original chromosome, leading to an unbalanced translocation. A cycle has a duplication on the host chromosome, while a bridge inserts the template copies into a deletion on the host chromosome.

Local-distant cluster A cluster of SVs that has both local rearrangements and rearrangements to other parts of the genome. 


\section{natureresearch}

\section{Reporting Summary}

Nature Research wishes to improve the reproducibility of the work that we publish. This form provides structure for consistency and transparency in reporting. For further information on Nature Research policies, see Authors \& Referees and the Editorial Policy Checklist.

\section{Statistics}

For all statistical analyses, confirm that the following items are present in the figure legend, table legend, main text, or Methods section.

n/a Confirmed

$\square$ \The exact sample size $(n)$ for each experimental group/condition, given as a discrete number and unit of measurement

Х $\square$ A statement on whether measurements were taken from distinct samples or whether the same sample was measured repeatedly

The statistical test(s) used AND whether they are one- or two-sided

Only common tests should be described solely by name; describe more complex techniques in the Methods section.

$\square$ \A description of all covariates tested

$\square$ \ A description of any assumptions or corrections, such as tests of normality and adjustment for multiple comparisons

$\square$ A full description of the statistical parameters including central tendency (e.g. means) or other basic estimates (e.g. regression coefficient)

AND variation (e.g. standard deviation) or associated estimates of uncertainty (e.g. confidence intervals)

$\square$ For null hypothesis testing, the test statistic (e.g. $F, t, r$ ) with confidence intervals, effect sizes, degrees of freedom and $P$ value noted

Give $P$ values as exact values whenever suitable.

\ For Bayesian analysis, information on the choice of priors and Markov chain Monte Carlo settings

Х $\square$ For hierarchical and complex designs, identification of the appropriate level for tests and full reporting of outcomes

$\square$ Estimates of effect sizes (e.g. Cohen's $d$, Pearson's $r$ ), indicating how they were calculated

Our web collection on statistics for biologists contains articles on many of the points above.

\section{Software and code}

Policy information about availability of computer code

Data collection Workflow Step Algorithm Version Dockstore Package*

WGS Alignment BWA-MEM v0.7.8-r455 https://goo.gl/oqp4Xd

EMBL/DKFZ SV caller DELLY v0.6.6 https://goo.gl/Y46MCo

EMBL/DKFZ SCNA caller ACEseq v1.0.189 https://goo.gl/4zoV42

EMBL/DKFZ SNV caller DKFZ somatic SNV workflow 1.0.132-1

EMBL/DKFZ indel caller Platypus v0.7.4

Sanger SCNA caller ascatNgs v1.5.2 https://goo.gl/9DSrbA

Sanger SV caller BRASS v4.012

grass v1.1.6

Sanger SNV caller CaVEMan v1.50

Sanger indel caller Pindel v1.5.7

Broad SCNA caller ABSOLUTE/JaBbA v1.5/? https://goo.gl/YkdtDt

Broad SV caller SvABA/dRanger/BreakPointer 2015-05-20/2016-03-13/2015-12-22

Broad SNV caller MuTect v1.1.4

Broad indel caller SvABA 2015-05-20

MUSE SNV caller MUSE v1.Orc https://goo.gl/5SR4bF

SMuFIN indel caller SMuFIN 2014-10-26 https://goo.gl/EuUP5k

Oxidative artefact filter OxOG 2016-4- $28 \mathrm{https://goo.gl/cUKP9K}$

SNV/Indel annotation VAGrENT v2.1.2 https://goo.gl/9DSrbA

ANNOVAR v2014Nov12 https://goo.gl/4zoV42

miniBAM generation VariantBAM v2017Dec12 https://goo.gl/S8h8e5

SNV/Indel merging and consensus generation SNV-MERGE V2017May26 https://goo.gl/TETSB8

SV merging and consensus generation SV-MERGE V2017Dec12 https://goo.gl/A9CEup

Strand bias filter DKFZ Strand Bias Filter v2016Dec15 https://goo.gl/8jXrvZ 
For manuscripts utilizing custom algorithms or software that are central to the research but not yet described in published literature, software must be made available to editors/reviewers. We strongly encourage code deposition in a community repository (e.g. GitHub). See the Nature Research guidelines for submitting code \& software for further information.

\section{Data}

Policy information about availability of data

All manuscripts must include a data availability statement. This statement should provide the following information, where applicable:

- Accession codes, unique identifiers, or web links for publicly available datasets

- A list of figures that have associated raw data

- A description of any restrictions on data availability

The PCAWG-generated alignments, variant calls, annotations and derived data sets are available for general research use for browsing and download at http:// dcc.icgc.org/pcawg/. In accordance with the data access policies of the ICGC and TCGA projects, most molecular, clinical and specimen data are in an open tier which does not require access approval. To access potentially identifying information, such as germline alleles and underlying read data, researchers will need to apply to the TCGA Data Access Committee (DAC) via dbGaP (https://dbgap.ncbi.nlm.nih.gov/aa/wga.cgi?page=login) for access to the TCGA portion of the data set, and to the ICGC Data Access Compliance Office (DACO; http://icgc.org/daco) for the ICGC portion.

\section{Field-specific reporting}

Please select the one below that is the best fit for your research. If you are not sure, read the appropriate sections before making your selection. $\bigotimes$ Life sciences Behavioural \& social sciences Ecological, evolutionary \& environmental sciences

For a reference copy of the document with all sections, see nature.com/documents/nr-reporting-summary-flat.pdf

\section{Life sciences study design}

All studies must disclose on these points even when the disclosure is negative.

Sample size

We compiled an inventory of matched tumour/normal whole cancer genomes in the ICGC Data Coordinating Centre. Most samples came from treatment-naïve, primary cancers, but there were a small number of donors with multiple samples of primary, metastatic and/or recurrent tumours. Our inclusion criteria were: (i) matched tumour and normal specimen pair; (ii) a minimal set of clinical fields; and (iii) characterisation of tumour and normal whole genomes using Illumina HiSeq paired-end sequencing reads.

We collected genome data from 2,834 donors, representing all ICGC and TCGA donors that met these criteria at the time of the final data freeze in autumn 2014

No formal power calculations were performed to decide sample size for PCAWG - we aggregated all genomes available at the time.

Data exclusions After quality assurance, data from 176 donors were excluded as unusable. Reasons for data exclusions included inadequate coverage, extreme bias in coverage across the genome, evidence for contamination in samples and excessive sequencing errors (for example, through 8oxoguanine). Data exclusion criteria were pre-established.

Replication In order to evaluate the performance of each of the mutation-calling pipelines and determine an integration strategy, we performed a largescale deep sequencing validation experiment. We selected a pilot set of 63 representative tumour/normal pairs, on which we ran the three core pipelines, together with a set of 10 additional somatic variant-calling pipelines contributed by members of the SNV Calling Working Group. To assess accuracy of SV calls, we therefore used the property that an SV must either generate a copy number change or be balanced, whereas artefactual calls will not respect this property. For individual SV callers, we estimated the true positive rate to be in the range $80-95 \%$ for samples in the pilot-63 dataset.

Randomization Not applicable - this was a descriptive study.

Blinding

Not applicable - this was a descriptive study.

\section{Reporting for specific materials, systems and methods}

We require information from authors about some types of materials, experimental systems and methods used in many studies. Here, indicate whether each material, system or method listed is relevant to your study. If you are not sure if a list item applies to your research, read the appropriate section before selecting a response. 


\begin{tabular}{l|l}
\hline n/a & Involved in the study \\
\hline & $\square$ Antibodies \\
$\square$ & $\square$ Eukaryotic cell lines \\
$\square$ & $\square$ Palaeontology \\
$\square$ & $\square$ Humals and other organisms \\
$\square$ & $\square$ Clinical data
\end{tabular}

\section{Human research participants}

Policy information about studies involving human research participants

Population characteristics manuscript). Demographically, the cohort included 1,469 males (55\%) and 1,189 females (45\%), with a mean age of 56 years (range, 1-90 years). Using population ancestry-differentiated single nucleotide polymorphisms (SNPs), the ancestry distribution was heavily weighted towards donors of European descent (77\% of total) followed by East Asians (16\%), as expected for large contributions from European, North American and Australian projects. We consolidated histopathology descriptions of the tumour samples, using the ICD-0-3 tumour site controlled vocabulary. Overall, the PCAWG data set comprises 38 distinct tumour types. While the most common tumour types are included in the dataset, their distribution does not match the relative population incidences, largely due to differences among contributing ICGC/TCGA groups in numbers sequenced.

Recruitment

Patients were recruited by the participating centres following local protocols. Samples obtained had to meet criteria on amount of tumour DNA available, meaning that the cohort is potentially somewhat biased towards larger tumours. Otherwise, we anticipate no major recruitment biases.

Ethics oversight
The Ethics oversight for the PCAWG protocol was undertaken by the TCGA Program Office and the Ethics and Governance Committee of the ICGC.

Note that full information on the approval of the study protocol must also be provided in the manuscript. 